\title{
HEAVY-ION FRACTIONATION IN THE IMPULSIVE SOLAR ENERGETIC PARTICLE EVENT OF 2002 AUGUST 20: ELEMENTS, ISOTOPES, AND INFERRED CHARGE STATES
}

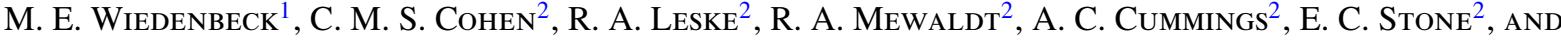 \\ T. T. VON ROSENVINGE ${ }^{3}$ \\ ${ }^{1}$ Jet Propulsion Laboratory, California Institute of Technology, Pasadena, CA 91109, USA; mark.e.wiedenbeck@jpl.nasa.gov \\ ${ }^{2}$ California Institute of Technology, Pasadena, CA 91125, USA \\ ${ }^{3}$ NASA Goddard Space Flight Center, Greenbelt, MD 20771, USA \\ Received 2009 November 16; accepted 2010 June 28; published 2010 July 27
}

\begin{abstract}
Measurements of heavy-ion elemental and isotopic composition in the energy range $\sim 12-60 \mathrm{MeV}$ nucleon ${ }^{-1}$ are reported from the Advanced Composition Explorer/Solar Isotope Spectrometer (ACE/SIS) instrument for the solar energetic particle (SEP) event of 2002 August 20. We investigate fractionation in this particularly intense impulsive event by examining the enhancements of elemental and isotopic abundance ratios relative to corresponding values in the solar wind. The elemental enhancement pattern is similar to those in other impulsive events detected by $A C E /$ SIS and in compilations of average impulsive-event composition. For individual elements, the abundance of a heavy isotope (mass $M_{2}$ ) is enhanced relative to that of a lighter isotope $\left(M_{1}\right)$ by a factor $\sim\left(M_{1} / M_{2}\right)^{\alpha}$ with $\alpha \simeq-15$. Previous studies have reported elemental abundance enhancements organized as a power law in $Q / M$, the ratio of estimated ionic charge to mass in the material being fractionated. We consider the possibility that a fractionation law of this form could be responsible for the isotopic fractionation as a power law in the mass ratio and then explore the implications it would have for the ionic charge states in the source material. Assuming that carbon is fully stripped $\left(Q_{\mathrm{C}}=6\right)$, we infer mean values of the ionic charge during the fractionation process, $Q_{Z}$, for a variety of elements with atomic numbers $7 \leqslant Z \leqslant 28$. We find that $Q_{\mathrm{Fe}} \simeq 21-22$, comparable to the highest observed values that have been reported at lower energies in impulsive SEP events from direct measurements near 1 AU. The inferred charge states as a function of $Z$ are characterized by several step increases in the number of attached electrons, $Z-Q_{Z}$. We discuss how this step structure, together with the known masses of the elements, might account for a variety of features in the observed pattern of elemental abundance enhancements. We also briefly consider alternative fractionation laws and the relationship between the charge states we infer in the source material and those derived from in situ observations.
\end{abstract}

Key words: Sun: abundances - Sun: flares - Sun: particle emission

\section{INTRODUCTION}

The class of solar energetic particle (SEP) events commonly designated as "impulsive" or as "3He-rich" is generally thought to involve particle acceleration powered by the release of magnetic energy as reconnection occurs in solar flares. The physical mechanism(s) primarily responsible for the conversion of magnetic energy to the kinetic energy of particles in the $\mathrm{MeV} /$ nucleon $^{-1}$ range has not been conclusively resolved. Particle intensities observed in these small events are characteristically much lower than in large, "gradual" SEP events, where shocks driven by coronal mass ejections (CMEs) in the solar corona and/or interplanetary medium are commonly believed to be responsible for the particle acceleration. The question of whether there exist "hybrid" events to which particles from both acceleration mechanisms contribute is still being debated (Cane et al. 2003). The problem of SEP acceleration has been discussed in a number of reviews (Reames 1995a, 1999; Aschwanden 2002; Klecker et al. 2006c; Mason 2007; von Rosenvinge \& Cane 2006).

Energetic particle composition, including elemental and isotopic abundances and ionic charge states, provides important observational constraints for distinguishing among particle acceleration models. Impulsive events have large enhancements of the ${ }^{3} \mathrm{He} /{ }^{4} \mathrm{He}$ ratio over the solar wind value of $5 \times 10^{-4}$, sometimes by factors $>10^{3}$. In addition, relative abundances of heavy elements (reviewed by Reames 1999; Mason 2007) show enhancements that increase with increasing difference in atomic number, $Z$. The $\mathrm{Fe} / \mathrm{O}$ ratio, which is often used in identifying impulsive events, is typically enhanced by a factor $\sim 10$, but lacks any strong correlation with the value of the ${ }^{3} \mathrm{He} /{ }^{4} \mathrm{He}$ enhancement.

Studies over the course of solar cycle 23 have confirmed and extended the earlier measurements, showing that the trend of increasing elemental abundance enhancements relative to coronal or solar wind values continues to the heaviest elements (Reames \& Ng 2004; Mason et al. 2004) and that the isotope ratios ${ }^{22} \mathrm{Ne} /{ }^{20} \mathrm{Ne}$ and ${ }^{26} \mathrm{Mg} /{ }^{24} \mathrm{Mg}$ tend to be enhanced by factors $\gtrsim 3$ (Dwyer et al. 2001; Wiedenbeck et al. 2003, 2008). Furthermore, at energies below $\sim 0.1 \mathrm{MeV}$ nucleon $^{-1}$, the ionic charge state of iron, $Q_{\mathrm{Fe}}^{\text {obs }}$, is comparable to solar wind values (Klecker et al. 2006b; DiFabio et al. 2008) but increases significantly between $\sim 0.1$ and $\sim 0.5 \mathrm{MeV}$ nucleon ${ }^{-1}$ (Klecker et al. 2006a, and references therein). (We use the superscript "obs" to designate charge state values observed in situ in order to distinguish them from values of $Q$ in the source material.) Statistical studies have found positive correlations among the abundance enhancements of heavy elements, the abundance enhancements of heavy isotopes, and observed iron charge states (Dwyer et al. 2001; Mason et al. 2004; Klecker et al. 2005; Möbius et al. 2000; DiFabio et al. 2008).

Most studies of heavy-ion composition in impulsive SEP events have been based on measurements made below a few $\mathrm{MeV}$ nucleon $^{-1}$. It is commonly found that energy spectra in these events soften at energies near an $\mathrm{MeV}$ nucleon $^{-1}$ (Mason et al. 2002b), and only a small fraction of the impulsive 
events observed below $1 \mathrm{MeV}$ nucleon ${ }^{-1}$ have been detected above $10 \mathrm{MeV}$ nucleon $^{-1}$. Furthermore, even when an event with enhanced ${ }^{3} \mathrm{He} /{ }^{4} \mathrm{He}$ is detected at these higher energies, intensities of high-energy $Z \geqslant 6$ ions are often too low to measure with present instrumentation. It is currently not known whether the impulsive SEP events that are detectable above $10 \mathrm{MeV}$ nucleon $^{-1}$ are just the most intense examples of the type of events studied at lower energies, or if an additional acceleration process is playing a role in producing these relatively rare events. The event of 2002 August 20, which is the focus of the present study, is an example of this type of event.

Wiedenbeck et al. $(2008,2009)$ examined elemental composition at high energies in several impulsive events and found significant deviations from a simple, monotonic increase of abundance enhancements with increasing $Z$. The same qualitative pattern of enhancements appeared in nearly all the events that were studied and was also found to be present in tables of typical impulsive SEP event abundances that have been compiled by several authors (Mason et al. 1986; Reames 1999; Mason et al. 2004). Large (factors of 3 or more), correlated enhancements in the isotopic abundance ratios ${ }^{22} \mathrm{Ne} /{ }^{20} \mathrm{Ne}$ and ${ }^{26} \mathrm{Mg} /{ }^{24} \mathrm{Mg}$ were also found in the high-energy impulsive events (Wiedenbeck et al. 2008, 2009), similar to the situation in gradual SEP events, but with significantly larger enhancements of the heavier isotopes (Leske et al. 2007).

Ionic charge states have long been recognized as an important probe of the conditions under which SEPs are produced (e.g., Sciambi et al. 1977; Ma Sung et al. 1981; Klecker et al. 1984), since they should provide indications of the temperature of a source plasma out of which the particles are accelerated and/ or the density of matter traversed when subsequent collisional stripping is important (Kartavykh et al. 2008, and references therein). However, while precise measurements of heavy-ion elemental and isotopic composition above $10 \mathrm{MeV}^{\text {nucleon }}{ }^{-1}$ have been made in a number of impulsive SEP events, measurements of ionic charge states have not been available at these energies. Space instrumentation for measuring charge states using electrostatic deflection (e.g., Möbius et al. 1998) has had neither the resolution nor the sensitivity to be applied above $\sim 1 \mathrm{MeV}$ nucleon $^{-1}$, and measurements using the Earth's magnetic field as a filter have required higher heavy-ion intensities than found in even the largest impulsive events studied to date.

One of the largest impulsive events observed at high energies during solar cycle 23 occurred on 2002 August 20 and was well measured at energies above $10 \mathrm{MeV}$ nucleon $^{-1}$ by the Solar Isotope Spectrometer (SIS) on the Advanced Composition Explorer (ACE) spacecraft, yielding possibly the most extensive set of elemental and isotopic composition observations that have been made in an individual impulsive SEP event. In this paper, we report those high-energy composition observations and compare with typical impulsive-event composition compiled based on lower-energy data. In addition, we use a combination of elemental and isotopic abundance enhancement data to infer mean ionic charge states of a number of elements during the fractionation process, using an indirect technique introduced by Cohen et al. (1999a, 1999b).

In Section 2, we report the ACE/SIS observations from the 2002 August 20 event and discuss the patterns of abundance enhancements relative to solar wind abundances. In Section 3, we discuss the use of these enhancements to infer ionic charge states and report charge states obtained assuming that the fractionation has a power-law dependence on $Q / M$. Section 4 addresses the possible role of these charge states in producing the

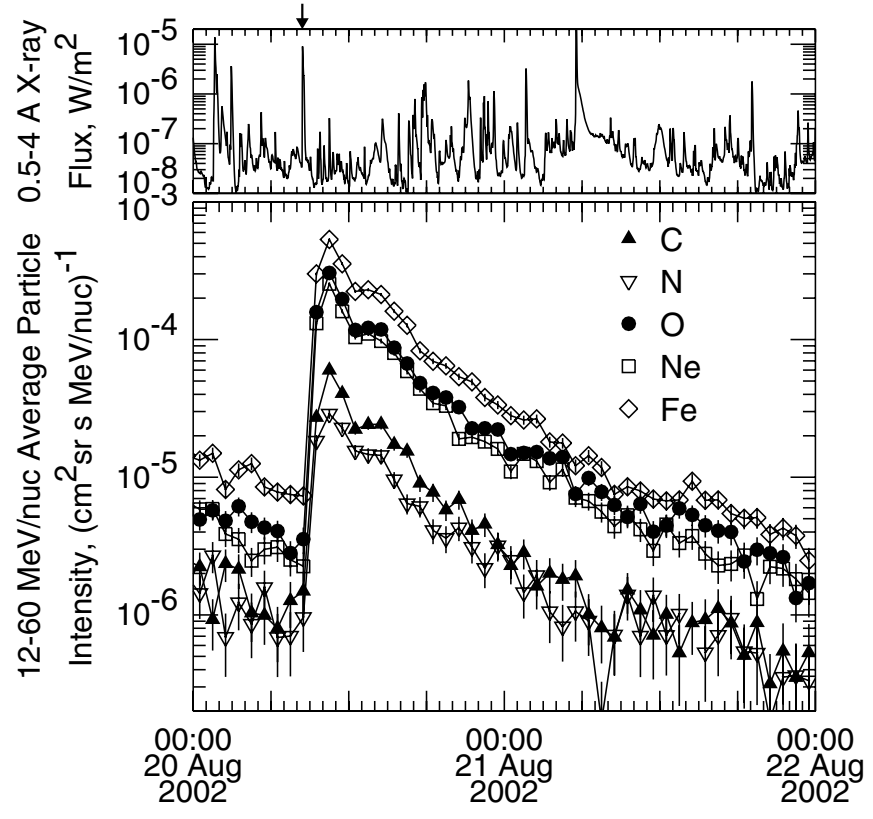

Figure 1. Lower panel: plot of hourly averaged intensity versus time for five heavy elements in the 2002 August 20 event. This event occurred following another relatively intense impulsive SEP event on 2002 August 19, but the earlier event had decayed sufficiently to allow accurate composition measurements for elements heavier than $\mathrm{He}$. Note the high value of $\mathrm{Fe} / \mathrm{O}$, which is characteristic of impulsive events, and the low intensity of $\mathrm{C}$ relative to $\mathrm{O}$ and $\mathrm{N}$ (in the solar wind $\mathrm{C}: \mathrm{N}: \mathrm{O}: \mathrm{Fe} \simeq 0.7: 0.08: 1: 0.12$ ). Upper panel: 5-minute average intensity of solar $x$-rays in the $0.5-4 \AA$ band from GOES. The M3.4 x-ray increase starting at 08:26 (arrow) from a flare at S10W38 was associated with the SEP event.

observed patterns of elemental and isotopic abundances. It also compares the charge states with those observed in other samples of matter from the Sun and with those expected in isothermal source plasmas. The section concludes with comparisons of our results for the 2002 August 20 event with findings from several previous SEP fractionation studies. Section 5 discusses relationships between this work and results of other impulsiveevent studies, and Section 6 summarizes the key results of the paper. The Appendix discusses a possible alternative form for the general fractionation law.

\section{OBSERVATIONS}

\subsection{Solar Flare Event}

As summarized by Leske et al. (2003), the SEP event of 2002 August 20 exhibited the characteristics commonly used in identifying impulsive events. As illustrated in the upper panel of Figure 1, it was associated with a moderate-intensity (M3.4), short-duration $(\sim 8$ minutes) $\mathrm{X}$-ray flare from an active region located at a western longitude well connected to the Earth by a nominal Parker-spiral magnetic field (AR 10079 at S10W38). In addition to the energetic ion event observed by $A C E / \mathrm{SIS}$, there was a strong type III radio burst and an energetic electron event exhibiting velocity dispersion. Because the ion event was particularly intense at high energies, it was also possible to observe velocity dispersion in the ion data collected with $A C E /$ SIS in spite of the relatively small spread in travel times $(\sim 1 \mathrm{hr})$ over the SIS energy range (Leske et al. 2003). The composition was characterized by an enhancement of $\mathrm{Fe} / \mathrm{O}$ by a factor $\sim 15$ and a high $e / p$ ratio. It was not possible to obtain a precise measurement of the ${ }^{3} \mathrm{He} /{ }^{4} \mathrm{He}$ ratio because the event occurred during the decay of another relatively intense impulsive event that occurred on the previous day. A small ${ }^{3} \mathrm{He}$ increase 


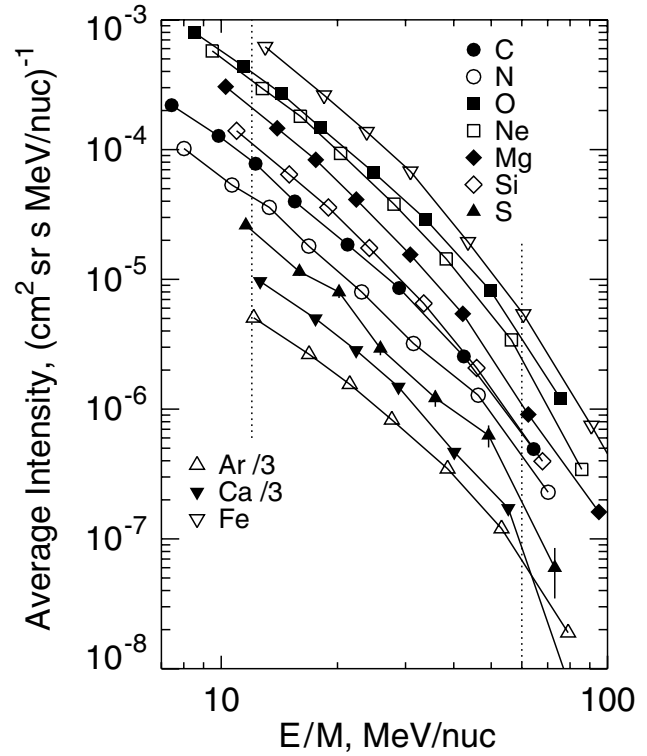

Figure 2. Measured energy spectra for 10 elements in the 2002 August 20 event. Abundances of these ten elements were obtained by integrating the spectra between 12 and $60 \mathrm{MeV}$ nucleon ${ }^{-1}$ (dotted lines). The average intensities plotted on the vertical axis are fluences divided by the $28 \mathrm{hr}$ interval (20 August 08:00 to 21 August 12:00) used for the study. Typical statistical uncertainties are illustrated by means of error bars included on the data points for sulfur.

superimposed on the decaying background was observed; for the heavier ions the increase above background was much larger (Figure 1). The estimated ${ }^{3} \mathrm{He} /{ }^{4} \mathrm{He}$ ratio for the 2002 August 20 event was no more than $\sim 0.1$. Background due to the event on 2002 August 19 also prevented the use of lower-energy data from the $A C E$ /ULEIS instrument in this study.

RHESSI images of the solar source region show soft-X-ray emission from a magnetic loop together with three hard-X-ray footpoints (S. Krucker 2008, private communication), a pattern that is seen in other events (Figure 10 of Dennis et al. 2007) and is interpreted as resulting from reconnection between the loop and an adjacent open field line. The event also had a small, faint CME (Leske et al. 2003).

\subsection{Elemental Composition}

The $A C E$ /SIS instrument (Stone et al. 1998a) used to obtain the elemental and isotopic composition data for this study employs two charged particle telescopes consisting of stacks of silicon solid-state detectors, the front two of which in each telescope are position sensitive, to determine the trajectories of incident particles and obtain multiple measurements of energy loss rates as particles slow down and stop in the instrument. Applying the widely used $d E / d x$ versus total energy technique, we are able to determine the atomic number, $Z$, and in some cases the mass, $M$, of detected particles. For particles stopping in a particular detector, and thus at a particular depth in the instrument, we obtain a measurement of the number of detected particles for each resolved element in an energy per nucleon interval that can be calculated for the particular $Z$ and stopping depth in the telescope, taking into account the distribution of entry directions into the instrument (Stone et al. 1998a, 1998b). Factors accounting for energy interval, livetime, readout efficiency, and geometrical acceptance are applied to convert the counts to particle intensities. Figure 2 shows the eventintegrated energy spectra obtained in this way for a number of elements. Because of the charge and mass dependence of the

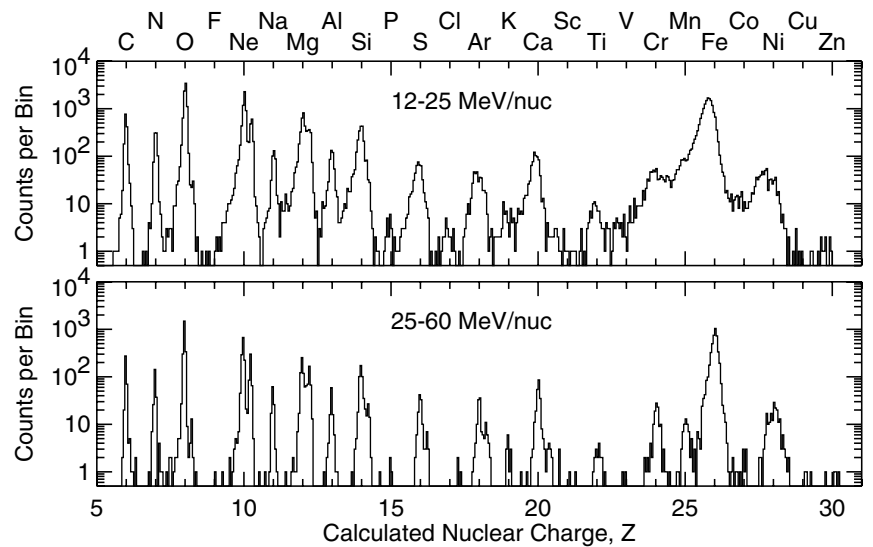

Figure 3. Histograms of calculated nuclear charge in two energy intervals. The structure that appears in the peaks for some charges (e.g., $\mathrm{Ne}$ ) is due to the presence of multiple isotopes. For elements that are well resolved in the $12-25 \mathrm{MeV}$ nucleon ${ }^{-1}$ interval (upper panel), abundance determinations use data from both energy intervals (see text for details). For elements that are not well separated in the $12-25 \mathrm{MeV}^{\text {nucleon }}{ }^{-1}$ interval, the abundance determination is based on just the $25-60 \mathrm{MeV}$ nucleon ${ }^{-1}$ data (lower panel) where the element resolution is better, but where counting statistics are more limited.

range-energy relation, the energy per nucleon interval covered is $Z$-dependent, with measurements for higher- $Z$ elements both starting and ending at higher energies than for lower- $Z$ elements. The spectral shapes are similar for all of the elements. The lowenergy portion of the spectra can be approximated as power laws with spectral indices $\sim-2.2 \pm 0.4$ at $12 \mathrm{MeV}_{\text {nucleon }}{ }^{-1}$ and softening significantly by $60 \mathrm{MeV}$ nucleon $^{-1}$. For the composition analysis, we integrated these spectra over the 12-60 $\mathrm{MeV}$ nucleon $^{-1}$ energy interval in which all elements from $\mathrm{C}$ through $\mathrm{Ni}$ can be measured, with at most minor extrapolation of the spectra near the end points.

For less-abundant elements, the statistical uncertainties on the individual intensity points are too large to obtain meaningful energy spectra. In these cases, we obtain abundances by comparing peak areas in charge histograms that combine data over a broad energy interval encompassing multiple stopping depths in the telescope. Figure 3 shows histograms for two energy intervals, 12-25 MeV nucleon ${ }^{-1}$ (upper panel) and 25-60 MeV nucleon ${ }^{-1}$ (lower panel). For the elements $\mathrm{Na}, \mathrm{Al}, \mathrm{P}, \mathrm{Cl}, \mathrm{Ni}$, and $\mathrm{Zn}$ that are well resolved in both of these energy intervals, we combined the data from the two histograms. Because the energy resolution of the instrument is better in the higher-energy interval, we restricted the analysis to the $25-60 \mathrm{MeV}^{\text {nucleon }}{ }^{-1}$ interval to obtain abundances for elements that are obscured by spillover from more-abundant neighboring elements in the 12-25 MeV nucleon ${ }^{-1}$ interval. This spillover results, in part, from the use of somewhat less-accurate pulse height measurements from the position-sensitive silicon detectors when analyzing high- $Z$ elements at low energies. Abundances for the elements $\mathrm{K}, \mathrm{Ti}, \mathrm{Cr}, \mathrm{Mn}$, and $\mathrm{Co}$ were obtained using the 25-60 MeV nucleon ${ }^{-1}$ interval, as were upper limits for F, Sc, $\mathrm{V}$, and $\mathrm{Cu}$. In using the histograms to derive elemental abundances, each particle was given a weight inversely proportional to the geometrical factor for its stopping depth in order to obtain abundances that should be directly comparable in terms of their energy weighting to those derived by integrating the energy spectra. Minor corrections for livetime and readout efficiency were made in a similar way.

Table 1 lists the results obtained for the elemental abundances in the 2002 August 20 event, together with uncertainties that 
Table 1

Elemental Composition in the 2002 August 20 SEP Event (Oxygen $\equiv 1.0)$

\begin{tabular}{|c|c|c|c|c|c|c|}
\hline Element & $Z$ & Counts & Method $^{\mathrm{a}}$ & $\begin{array}{c}\text { SEP } \\
\text { Abundance }^{b}\end{array}$ & $\begin{array}{c}\text { SW } \\
\text { Abundance }^{c}\end{array}$ & $\begin{array}{c}\text { Enhancement } \\
\text { (SEP/SW) }\end{array}$ \\
\hline$\overline{\mathrm{C}}$ & 6 & 1697 & 1 & $0.192 \pm 0.009$ & $0.68 \pm 0.07$ & $0.28 \pm 0.03$ \\
\hline $\mathrm{N}$ & 7 & 966 & 1 & $0.113 \pm 0.006$ & $0.078 \pm 0.005$ & $1.45 \pm 0.12$ \\
\hline $\mathrm{O}$ & 8 & 9341 & 1 & $1.00 \pm 0.04$ & $1.00 \pm 0.07$ & $1.00 \pm 0.08$ \\
\hline $\mathrm{F}$ & 9 & 2 & 3 & $<0.0017$ & $\ldots$ & \\
\hline $\mathrm{Ne}$ & 10 & 7931 & 1 & $0.84 \pm 0.03$ & $0.14 \pm 0.03$ & $6.0 \pm 1.3$ \\
\hline $\mathrm{Na}$ & 11 & 528 & 2 & $0.0505 \pm 0.0030$ & $0.0090 \pm 0.0015$ & $5.6 \pm 1.0$ \\
\hline $\mathrm{Mg}$ & 12 & 4641 & 1 & $0.517 \pm 0.022$ & $0.15 \pm 0.05$ & $3.5 \pm 1.2$ \\
\hline $\mathrm{Al}$ & 13 & 609 & 2 & $0.057 \pm 0.003$ & $0.012 \pm 0.003$ & $4.8 \pm 1.2$ \\
\hline $\mathrm{Si}$ & 14 & 2476 & 1 & $0.252 \pm 0.011$ & $0.14 \pm 0.05$ & $1.8 \pm 0.6$ \\
\hline$P$ & 15 & 25 & 2 & $0.0023 \pm 0.0005$ & $\ldots$ & $\ldots$ \\
\hline$S$ & 16 & 557 & 1 & $0.056 \pm 0.003$ & $0.050 \pm 0.015$ & $1.1 \pm 0.3$ \\
\hline $\mathrm{Cl}$ & 17 & 26 & 2 & $0.0024 \pm 0.0005$ & $\ldots$ & $\ldots$ \\
\hline $\mathrm{Ar}$ & 18 & 446 & 1 & $0.0452 \pm 0.0028$ & $0.0031 \pm 0.0008$ & 15. \pm 4 \\
\hline $\mathrm{K}$ & 19 & 13 & 3 & $0.0053 \pm 0.0016$ & $\ldots$ & $\ldots$ \\
\hline $\mathrm{Ca}$ & 20 & 878 & 1 & $0.089 \pm 0.005$ & $0.0081 \pm 0.0015$ & $11.0 \pm 2.1$ \\
\hline $\mathrm{Sc}$ & 21 & 5 & 3 & $<0.0030$ & $\ldots$ & $\ldots$ \\
\hline $\mathrm{Ti}$ & 22 & 17 & 3 & $0.0068 \pm 0.0018$ & $\ldots$ & $\ldots$ \\
\hline $\mathrm{V}$ & 23 & 2 & 3 & $<0.0014$ & $\ldots$ & $\ldots$ \\
\hline $\mathrm{Cr}$ & 24 & 117 & 3 & $0.047 \pm 0.006$ & $0.0020 \pm 0.0003$ & $24 . \pm 5$ \\
\hline $\mathrm{Mn}$ & 25 & 60 & 3 & $0.024 \pm 0.004$ & $\ldots$ & $\ldots$ \\
\hline $\mathrm{Fe}$ & 26 & 17807 & 1 & $1.79 \pm 0.07$ & $0.12 \pm 0.05$ & 15. \pm 6 \\
\hline $\mathrm{Co}$ & 27 & 10 & 3 & $0.0040 \pm 0.0013$ & $\ldots$ & $\ldots$ \\
\hline $\mathrm{Ni}$ & 28 & 873 & 2 & $0.081 \pm 0.004$ & $0.007 \pm 0.003$ & 12. \pm 6 \\
\hline $\mathrm{Cu}$ & 29 & 3 & 3 & $<0.0020$ & $\ldots$ & $\ldots$ \\
\hline $\mathrm{Zn}$ & 30 & 13 & 2 & $0.0012 \pm 0.0003$ & $\ldots$ & $\ldots$ \\
\hline
\end{tabular}

Notes.

a Abundance determination methods: 1 -spectrum integration, $12-60 \mathrm{MeV}^{\text {nucleon }}{ }^{-1}$; 2 - weighted counts histogram, $12-60 \mathrm{MeV}$ nucleon ${ }^{-1}$; 3-weighted counts histogram, 25-60 $\mathrm{MeV}$ nucleon $^{-1}$.

${ }^{\mathrm{b}}$ Uncertainties include both statistical and estimated systematic contributions (see the text).

c Bochsler (2007) and Karrer et al. (2007).

include both the statistical and the systematic contributions. The systematic uncertainties, estimated to be $\sim 4 \%-8 \%$, were determined by comparing abundances derived using the different approaches in cases where that was possible. Also indicated is the method used for deriving each value (see above) and the number of events that were used, which gives an indication of the statistical contribution to the uncertainty. Composition measured in the solar wind (see compilation of Bochsler 2007; Karrer et al. 2007) is shown for comparison as are the SEP abundance enhancements (SEP/SW) for cases where solar wind (SW) values are available. The uncertainties given for the enhancement factors include contributions from both the SEP and the solar wind values. Figure 4 displays the elemental abundance enhancement factors as a function of $Z$.

Also plotted in Figure 4 are average abundance enhancements compiled from studies of sizeable numbers of impulsive SEP events at energies lower than those used in the present investigation: $0.32-0.45 \mathrm{MeV}$ nucleon $^{-1}$ from Mason et al. (2004, diamonds), $\sim 1-3 \mathrm{MeV}$ nucleon $^{-1}$ from Mason et al. (1986, open circles), and 1.9-2.8 MeV nucleon ${ }^{-1}$ from Reames (1995a, 1999, rectangles with heights representing the rms variation among the observed events). There is reasonable agreement between the qualitative pattern of abundance enhancements found in the 2002 August 20 event and in the various compilations of average impulsive SEP abundances; for some elements there is good quantitative agreement as well. It should be noted that we have computed enhancement factors from the published abundance compilations employing the same solar wind values used for normalizing our data (Table 1). Thus differences among the

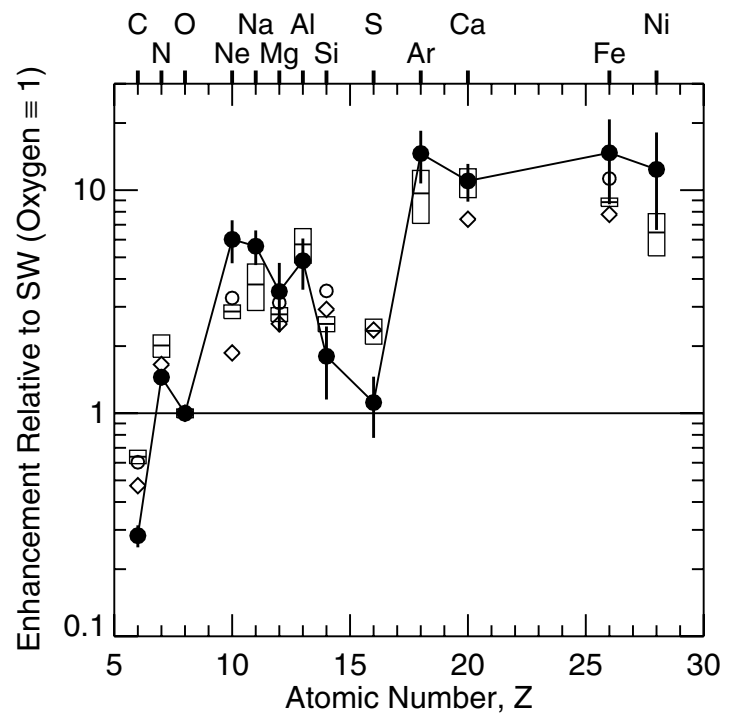

Figure 4. Elemental abundance enhancements (filled circles, normalized to 1 at oxygen) in the 2002 August 20 impulsive event relative to solar wind values (Bochsler 2007; Karrer et al. 2007). Also shown are average impulsive-event abundances tabulated by Mason et al. (1986) (open circles), Mason et al. (2004) (diamonds), and Reames (1999) (rectangles) normalized to the same solar wind values. For the Reames (1999) points, the horizontal bar inside the rectangle indicates the mean value and the height of the rectangle shows the $\pm 1 \sigma$ variation among the events studied. Many of the details of the average abundance pattern are present in the 2002 August 20 event, as well as in other individual events (Wiedenbeck et al. 2008, 2009). 


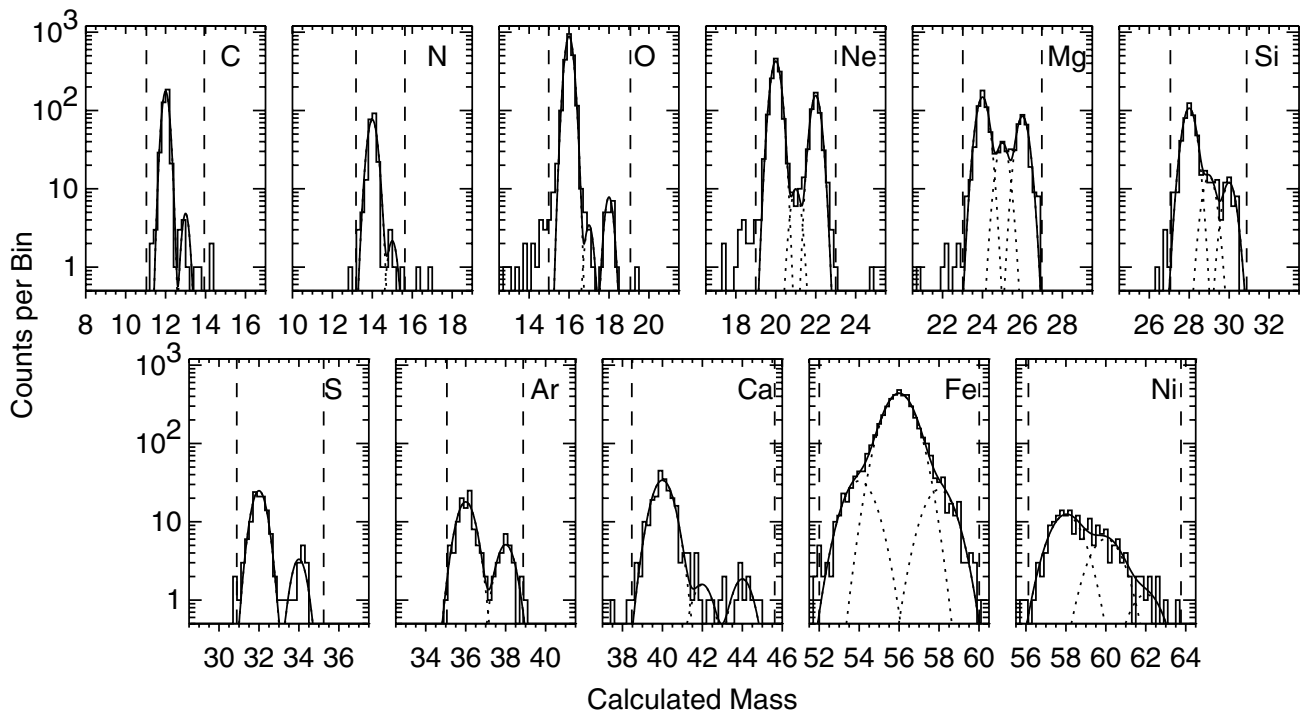

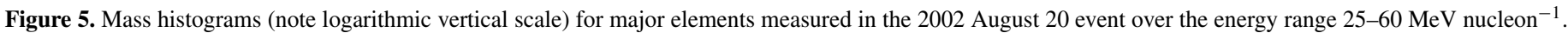

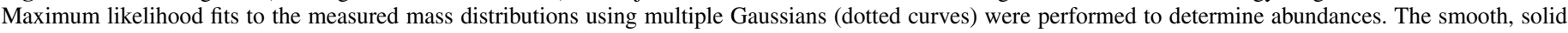
curves show the sums of the fitted Gaussians. The fits were restricted to the mass regions between the vertical dashed lines.

various data sets reflect only differences among the SEP values. Until recently, the solar wind composition data were not sufficiently complete to be used as a normalization for this large set of elements and "SEP-derived coronal abundances" were used as proxies for actual coronal abundances. Wiedenbeck et al. (2008) showed composition measured in a number of impulsive SEP events normalized both to solar wind and to SEP-derived coronal values. Although the general pattern of derived enhancements was similar, in some individual cases (e.g., N/C) the choice of normalization made a significant difference in the enhancement results. Although elemental enhancements in impulsive events measured at energies of a few $\mathrm{MeV}$ nucleon $^{-1}$ and below are typically ordered by $Z$ (or, equivalently, by mass) from $\mathrm{C}$ to $\mathrm{Fe}$ and beyond (Mason et al. 2004), it is clear from Figure 4 that there are sizeable, reproducible deviations from a simple monotonic increase. This pattern can also be seen in the abundances derived by Slocum et al. (2003) for a sample of SEP events selected to have ${ }^{3} \mathrm{He} /{ }^{4} \mathrm{He}>0.1$.

The enhancement pattern includes: a peak at $\mathrm{N}$, which is more enhanced than either of its neighboring elements, $\mathrm{C}$ and $\mathrm{O}$; a large increase between $\mathrm{O}$ and $\mathrm{Ne}$; a gradual decline from $\mathrm{Ne}$ through $\mathrm{S}$, interrupted by a small peak at $\mathrm{Al}$; a second large increase between $\mathrm{S}$ and $\mathrm{Ar}$; and a plateau from $\mathrm{Ar}$ through Ni. Wiedenbeck et al. (2008) showed that these features are observed in most of the impulsive events that have been measured with $A C E /$ SIS and had sufficient intensity to provide statistically significant abundance determinations for this set of elements.

\subsection{Isotopic Composition}

For the more-abundant elements, it has also been possible to obtain data on isotopic composition. The isotope ratios ${ }^{22} \mathrm{Ne} /$ ${ }^{20} \mathrm{Ne}$ and ${ }^{26} \mathrm{Mg} /{ }^{24} \mathrm{Mg}$, which exhibit correlated enhancements, have been shown for a number of impulsive SEP events by Wiedenbeck et al. (2008, 2009). The 2002 August 20 event is unique among the impulsive SEP events measured by $A C E / \mathrm{SIS}$ during solar cycle 23 in that it provided a large enough sample of high-energy heavy ions to enable investigations of numerous additional isotope ratios. Figure 5 shows mass histograms for 11 elements in this event. Since, as noted in our discussion of the charge histograms in Figure 3, the instrument's charge (and mass) resolution is better for particles that penetrate deep enough into the detector stack so that it is not necessary to use pulse heights from the position-sensitive silicon detectors in deriving $d E / d x$, we have restricted the isotopic composition analysis to the interval $25-60 \mathrm{MeV}$ nucleon ${ }^{-1}$ where essentially all the data for the elements $\mathrm{C}$ through $\mathrm{Ni}$ come from particles that meet this penetration-depth criterion.

The relative numbers of counts for each isotope in the mass histograms were derived by means of maximum likelihood fits of multiple Gaussian peaks, as indicated by the smooth curves overplotted on the histograms. In addition to fitting the peak areas, we adjusted three other parameters: the rms width of the peaks, the center position of the dominant isotope peak, and the spacing between adjacent peaks. Distributions plotted in Figure 5 (both measured and fitted) have been corrected using the fitted values of the latter two of these parameters. Measured mass peaks tend to have non-Gaussian tails that can be important when fitting rare isotopes having abundances that are only a few percent of that of an adjacent major isotope; we have no general description of the shapes of the tails that could be used to improve the fits. Tails on the low-mass (or low-charge) sides of the peaks are particularly prominent (see the $\mathrm{O}$ and $\mathrm{Ne}$ mass histograms in Figure 5 and the Fe peak in Figure 3). Fortunately, only one isotope ratio is thought to be strongly affected by a lowmass tail, ${ }^{54} \mathrm{Fe} /{ }^{56} \mathrm{Fe}$.

The selection of events to be included in the mass histogram for an element involves a cut requiring that the particle energy be in the interval $25-60 \mathrm{MeV}^{\text {nucleon }}{ }^{-1}$. However, when this cut is made the particle masses are not known, and even after fitting the mass distributions one does not obtain masses of individual particles. For purposes of making the energy per nucleon cut, we assume that all the particles corresponding to a given element have the mass of the dominant isotope of that element. This introduces a bias in the mass distribution since we are actually making a selection based on total energy, not on energy per nucleon. To correct for this bias, we have performed 
Table 2

Isotopic Composition in the 2002 August 20 SEP Event

\begin{tabular}{lccc}
\hline \hline $\begin{array}{c}\text { Isotope } \\
\text { Ratio }\end{array}$ & SEP Value & SW Value & $\begin{array}{c}\text { Enhancement } \\
(\text { SEP/SW })\end{array}$ \\
\hline${ }^{13} \mathrm{C} /{ }^{12} \mathrm{C}$ & $0.024(+0.009,-0.007)$ & 0.0112 & $2.1(+0.8,-0.6)$ \\
${ }^{15} \mathrm{~N} /{ }^{14} \mathrm{~N}$ & $0.020(+0.012,-0.009)$ & $0.0050 \pm 0.0014$ & $4.0 \pm 2.7$ \\
${ }^{18} \mathrm{O} /{ }^{16} \mathrm{O}$ & $0.0077 \pm 0.0019$ & $0.0022 \pm 0.0005$ & $3.5 \pm 1.1$ \\
${ }^{22} \mathrm{Ne} /{ }^{20} \mathrm{Ne}$ & $0.321 \pm 0.018$ & $0.0730 \pm 0.0016$ & $4.39 \pm 0.26$ \\
${ }^{25} \mathrm{Mg} /{ }^{24} \mathrm{Mg}$ & $0.229 \pm 0.026$ & $0.130 \pm 0.007$ & $1.77 \pm 0.22$ \\
${ }^{26} \mathrm{Mg} /{ }^{24} \mathrm{Mg}$ & $0.50 \pm 0.04$ & $0.144 \pm 0.009$ & $3.5 \pm 0.3$ \\
${ }^{29} \mathrm{Si} /{ }^{28} \mathrm{Si}$ & $0.119 \pm 0.021$ & $0.0490 \pm 0.0022$ & $2.4 \pm 0.4$ \\
${ }^{30} \mathrm{Si} /{ }^{28} \mathrm{Si}$ & $0.099 \pm 0.017$ & $0.0354 \pm 0.0028$ & $2.8 \pm 0.5$ \\
${ }^{34} \mathrm{~S} /{ }^{32} \mathrm{~S}$ & $0.118(+0.037,-0.030)$ & $0.045 \pm 0.010$ & $2.6 \pm 1.0$ \\
${ }^{38} \mathrm{Ar} /{ }^{36} \mathrm{Ar}$ & $0.25 \pm 0.06$ & $0.189 \pm 0.011$ & $1.3 \pm 0.3$ \\
${ }^{44} \mathrm{Ca} /{ }^{40} \mathrm{Ca}$ & $0.048 \pm 0.017$ & $0.021 \pm 0.004$ & $2.3 \pm 0.9$ \\
${ }^{54} \mathrm{Fe} /{ }^{56} \mathrm{Fe}$ & $0.067(+0.009,-0.007)$ & $0.068 \pm 0.004$ & $0.99 \pm 0.14$ \\
${ }^{60} \mathrm{Ni} /{ }^{58} \mathrm{Ni}$ & $0.44 \pm 0.12$ & $0.25 \pm 0.07$ & $1.7 \pm 0.7$ \\
\hline
\end{tabular}

Notes. ${ }^{\text {a }}$ Solar wind values from Bochsler (2007), except for ${ }^{13} \mathrm{C} /{ }^{12} \mathrm{C}$, which was assumed to have the solar system value given by Lodders (2003).

Monte Carlo simulations in which we assumed an isotropic population of particles with the measured energy spectrum (Figure 2, assumed to have the same shape for all the isotopes of an element) and determined the relative numbers of particles of each isotope collected in the specified energy interval, in the same way as was done with the actual data. These numbers were then used to correct the relative numbers of counts obtained by fitting the mass histograms. The corrections increased the relative abundance value for the more massive isotope by amounts ranging between $4 \%$ per mass unit separation for $\mathrm{Ni}$ and $18 \%$ for $\mathrm{C}$.

Corrections to the measured isotope ratios were also made to account for contributions of galactic cosmic rays (GCRs) to the isotope intensities in the $25-60 \mathrm{MeV}$ nucleon $^{-1}$ energy interval that was used. The isotopes ${ }^{15} \mathrm{~N},{ }^{13} \mathrm{C}$, and ${ }^{12} \mathrm{C}$ had the largest GCR contributions, amounting to $20 \%, 5 \%$, and $2 \%$, respectively. For all other isotopes, the contributions were $<1 \%$.

Table 2 shows the corrected isotope ratios in the 25-60 MeV nucleon ${ }^{-1}$ energy interval for the 2002 August 20 event, together with the corresponding solar wind values from Bochsler (2007) and the isotopic enhancement factors obtained by taking the SEP/SW ratio. In the case of ${ }^{13} \mathrm{C} /$ ${ }^{12} \mathrm{C}$, for which we do not have a published solar wind value, we have used the terrestrial value from Lodders (2003). This value is generally consistent with values derived for the solar photosphere using spectral lines of CO (Scott et al. 2006) and with values found in other solar system bodies (Woods \& Willacy 2009; Woods 2009). A preliminary report of carbon isotope observations in the solar wind (Koeten et al. 2008; R. F. Wimmer-Schweingrüber 2008, private communication) from $A C E /$ SWICS suggests agreement with the terrestrial value, within the relatively large uncertainty of the solar wind measurement. In calculating the uncertainty in the ${ }^{13} \mathrm{C} /{ }^{12} \mathrm{C}$ enhancement factor, we have not attempted to include a contribution attributable to an uncertainty in the solar wind value. Figure 6 contains a log-log plot showing these enhancement factors as a function of the ratio of masses between the two isotopes. Enhancement factors derived from mass distribution fits in which there is not even an inflection point separating an isotope from one of its neighbors $\left({ }^{29} \mathrm{Si},{ }^{54} \mathrm{Fe}\right.$, and ${ }^{60} \mathrm{Ni}$, shown with unfilled points in Figure 6) could be significantly affected by spillover from the adjacent isotope, particularly in the case of ${ }^{54} \mathrm{Fe}$, as noted above.

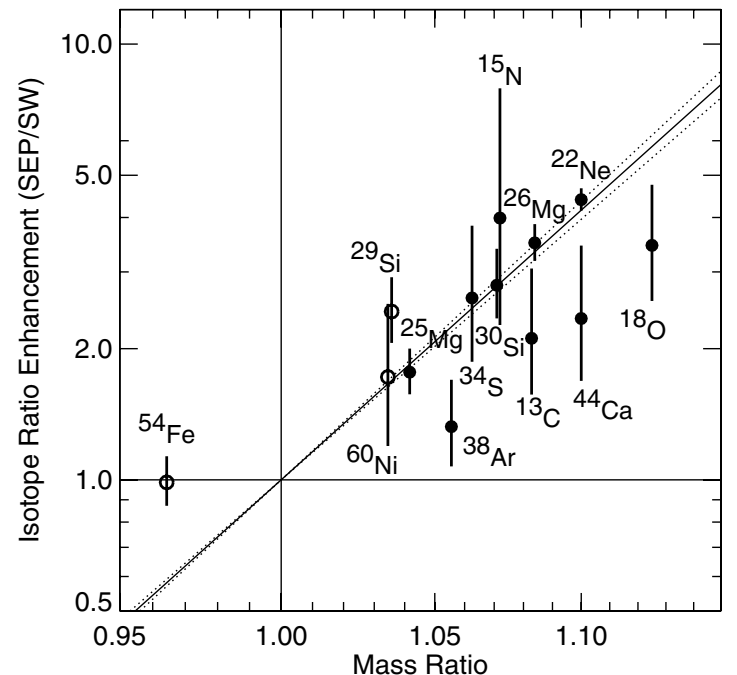

Figure 6. Log- $\log$ plot of isotope enhancements in the 2002 August 20 event relative to solar wind values as a function of the ratio of isotope masses. Each point is labeled with the mass number of the isotope in the numerator of the ratio. In all cases the dominant isotope of the same element was used for the denominator (i.e., ${ }^{12} \mathrm{C},{ }^{14} \mathrm{~N},{ }^{16} \mathrm{O},{ }^{20} \mathrm{Ne},{ }^{24} \mathrm{Mg},{ }^{28} \mathrm{Si},{ }^{32} \mathrm{~S},{ }^{36} \mathrm{Ar},{ }^{40} \mathrm{Ca},{ }^{56} \mathrm{Fe}$, and ${ }^{58} \mathrm{Ni}$ ). Error bars include uncertainties (statistical and systematic, see text) in the SEP measurement as well as uncertainties in the solar wind values. The diagonal solid and dotted lines indicate the least squares fit of a power law to the data and the effect of a $\pm 1 \sigma$ uncertainty in the fitted exponent. Only those data points shown as filled circles, which correspond to isotopes separated by at least an inflection point in the fitted distribution (Figure 5), were included in the fit. Note that essentially the same power-law slope can be obtained from the enhancement of ${ }^{22} \mathrm{Ne} /{ }^{20} \mathrm{Ne}$ alone.

The plot in Figure 6 shows a general trend of increasing relative enhancement with increasing mass ratio. This trend encompasses isotope ratios obtained from elements ranging from $\mathrm{C}$ to $\mathrm{Ni}$ and appears largely unaffected by the $Z$ values corresponding to the individual points. Assuming that the mass fractionation goes as a power law in the mass ratio, we have fit a straight line through the origin (mass ratio $=1$, enhancement $=$ 1) to the filled points in Figure 6. We obtain a best-fit slope and associated uncertainty of $15.0 \pm 0.5$, as indicated by solid and dotted lines in the figure. It should be noted that a fractionation 
line can be determined from the enhancement factor for a single isotope ratio and that ${ }^{22} \mathrm{Ne} /{ }^{20} \mathrm{Ne}$, which has one of the larger mass ratios as well as the smallest uncertainty in the derived enhancement, is particularly useful for this purpose. Using only the ${ }^{22} \mathrm{Ne} /{ }^{20} \mathrm{Ne}$ ratio, one would obtain a slope of $15.5 \pm 0.6$. By using the ${ }^{22} \mathrm{Ne} /{ }^{20} \mathrm{Ne}$ ratio alone, or sometimes in combination with ${ }^{26} \mathrm{Mg} /{ }^{24} \mathrm{Mg}$, it is possible to derive the mass fractionation exponent in some smaller impulsive SEP events where most of the isotope ratios included in Figure 6 are unavailable due to limited counting statistics.

In many environments where isotopic abundances are studied (e.g., in many terrestrial and meteoritic samples), one finds that the relative excesses or depletions of the isotopes of an element are proportional to the mass difference. That result is to be expected under conditions where the fractionation is relatively weak since the linear dependence on $M$ is merely the lowest order term in the Taylor series expansion of a more general mass dependence where the higher order terms are negligible for mass differences of just a few mass units. However, for the mass fractionation observed in the 2002 August 20 impulsive SEP event, as well as in other impulsive events (Wiedenbeck et al. 2003, 2009), the mass dependence of the fractionation is strong enough that the linear approximation is not applicable. With a $M^{15}$ mass dependence, the ratio of the ${ }^{25} \mathrm{Mg} /{ }^{24} \mathrm{Mg}$ excess to that of ${ }^{26} \mathrm{Mg} /{ }^{24} \mathrm{Mg}$ is $\left.R=((25 / 24))^{15}-1\right) /\left((26 / 24)^{15}-1\right)=0.36$ (see Figure 6) rather than the value of 0.5 that one would expect with a weak dependence of the fractionation on mass. For some impulsive events, fractionation exponents as large as 25 have been reported (Wiedenbeck et al. 2009), which would give $R=0.28$.

\section{INFERRED CHARGE STATES}

\subsection{Basic Scenario}

The elemental and isotopic composition in the 2002 August 20 event, as presented in Section 2, is characterized by large, systematic differences from the composition of the solar wind, which we have adopted as a proxy for abundances in the solar corona, the assumed source of material from which SEPs are derived. In this and the following sections, we consider the origin of the composition differences and discuss the information they may provide about conditions in the source and about the processes involved in producing the SEPs from it. It should be emphasized that we are not assuming that the material from which impulsive SEPs are derived has the properties of the solar wind other than its elemental and isotopic composition. In particular, we are not assuming that it has solar-wind-like ionic charge state distributions. In Section 3.2, we use the SEP fractionation pattern to infer mean charge states and in Section 4 we compare those inferred charge states with values found in the solar wind and in other samples of solar material to investigate their origin.

To set a framework for this discussion, we will characterize the processes involved in going from the coronal source to the SEPs observed in interplanetary space as follows.

1. Fractionation. The modification of the relative elemental and isotopic abundances of different ion species. We assume that the fractionation depends on the ionic charge $Q$ and the mass $M$ of the ions.

2. Acceleration. The energization of the ions into the $\mathrm{MeV}$ nucleon ${ }^{-1}$ range. This is assumed to take place with little or no alteration of the relative abundances of the ion species being accelerated and without changing the ionic charge states.

3. Stripping. The alteration of the ionic charge states of various ion species. This is thought to occur primarily by Coulomb collisions with the matter being traversed.

Considering fractionation, acceleration, and stripping as distinct processes that take place sequentially in some order is, at best, an approximation to more complex situations in which changes in elemental and isotopic abundances, charge states, and energy may all be occurring simultaneously. However, this simplification seems appropriate as a first approximation to these more complex situations, and it has the advantage that it allows inferences to be drawn about the relative sequence of the various processes. The applicability of the assumptions should be checked when applying our results to specific situations.

In the following sections, we use the observed elemental and isotopic composition together with assumptions about the $Q$ and $M$ dependence of the fractionation process to obtain information about the state of the material upon which it is acting (e.g., the ionic charge states that are present and, if the source material is in thermal equilibrium, the temperature to which these charge states correspond). It should be kept in mind that the ionic charge states that play a role in determining the amount of fractionation need not be the same as the charge states present in the SEPs that can be observed in interplanetary space. If the fractionation occurs before acceleration and stripping, one would expect the relevant charge states to be those present in a sample of coronal plasma upon which the fractionation acts. Stripping may subsequently alter the ionic charges. On the other hand, if the fractionation were to take place after acceleration and stripping have occurred, then the charge states controlling the fractionation would more closely correspond to those that can be observed in situ, after taking into account the effects the fractionation process itself may have on charge state distributions. Thus, by comparing the charge states needed to account for the fractionation with those present in the SEPs that escape from the Sun, it may be possible to address the question of whether the fractionation occurs before or after the stripping.

\subsection{Fractionation Dependent upon $Q / M$}

Breneman \& Stone (1985) found that elemental abundance enhancements in large, gradual SEP events tend to be organized as a power law in $Q / M$, with the fractionation exponent varying from event to event (see also Garrard \& Stone 1994). For that work, average ionic charge values measured in a number of gradual events at lower energies were used, since no direct measurements of $Q$ were available in the events that were being studied. This $Q / M$ fractionation is thought to be the source of the mass-dependent fractionation of isotopic composition (Leske et al. 2007, and references therein) in gradual events. Since it is likely that the isotopes of a given element have the same charge state distribution, isotope ratio enhancements are expected to be insensitive to $Q$. In a similar way, $Q / M$ fractionation is thought to be responsible for the observed correlation between enhancements of ${ }^{22} \mathrm{Ne} /{ }^{20} \mathrm{Ne}$ and $\mathrm{Na} / \mathrm{Mg}$ if, as expected, $\mathrm{Na}$ and $\mathrm{Mg}$ tend to have the same charge states ( +9 and +10 , respectively) in most gradual events (Cohen et al. 1999a; Leske et al. 2007).

In recent investigations of heavy element composition in impulsive SEP events (Slocum et al. 2003; Mason et al. 2004; Reames \& Ng 2004), it was shown that abundance enhancements are reasonably well organized as power laws in 
$Q / M$ if one assumes equilibrium charge states corresponding to a temperature $\sim 3 \mathrm{MK}$. As with the gradual SEP event fractionation studies mentioned above, direct measurements of $Q$ were not available in most of the events.

Motivated by these results indicating that abundance enhancements are organized as power laws in $Q / M$ in both gradual and impulsive SEP events and by the finding that mass fractionation in impulsive events is organized as a power law in the mass (Leske et al. 2007; Wiedenbeck et al. 2009, and discussion in Section 2.3), we have considered the hypothesis that elemental and isotopic enhancements that we find in impulsive events could also be due to a mechanism that leads to fractionation of the form

$$
\mathcal{E}_{i}=\left(Q_{i} / M_{i}\right)^{\alpha},
$$

where $\mathcal{E}_{i}$ represents the enhancement of ions with charge $Q_{i}$ and mass $M_{i}$ relative to their abundances in a population of seed material. We assume this seed population to have the elemental and isotopic abundances found in solar coronal material and use solar wind measurements as a proxy for this composition. The fractionation exponent, $\alpha$, may vary from event to event.

In studying composition in several large SEP events that occurred in 1997 and 1998, Cohen et al. (1999a, 1999b) noted that if the fractionation has the form of a power law in $Q / M$, then a determination of the enhancement of an isotope ratio such as ${ }^{22} \mathrm{Ne} /{ }^{20} \mathrm{Ne}$ is sufficient for calculating the power-law exponent and that the use of this exponent along with elemental composition allows one to infer ratios of ionic charge states. We have applied this technique to infer ionic charge states in the 2002 August 20 impulsive event. Applying Equation (1) to an element $Z$ for which the ionic charge is to be derived and to a reference element $Z_{0}$ for which the ionic charge is known, one obtains

$$
Q_{Z}=Q_{Z_{0}} \frac{M_{Z}}{M_{Z_{0}}}\left(\frac{\mathcal{E}_{Z}}{\mathcal{E}_{Z_{0}}}\right)^{1 / \alpha} \quad \text { (for }[Q / M]^{\alpha} \text { fractionation). }
$$

Here, the values of $M_{Z}$ and $Q_{Z}$ are taken to be those characteristic of the element with atomic number $Z$. We use carbon $(Z=6)$ for the normalization, taking $M_{\mathrm{C}}=12$ and assuming $Q_{\mathrm{C}}=6.0$. According to the tables of Mazzotta et al. (1998), an isothermal plasma will have $Q_{\mathrm{C}} \simeq 5.89$ at $2 \mathrm{MK}$ and 5.96 at $2.5 \mathrm{MK}$. For the 2002 August 20 event, $\alpha=-15.0 \pm 0.5$, the negative of the mass fractionation exponent (Section 2.3) because we are expressing the fractionation as a power law in $Q / M$ rather than in $M / Q$. The elemental abundance enhancements were obtained from Table 1.

The open points in Figure 7 show the charge states derived using this procedure, plotted in the form $Z-Q_{Z}$ (number of electrons attached) versus $Z$. The error bars include the effects of statistical and systematic uncertainties in the SIS elemental abundances and in the solar wind values used for normalization. Also included is the uncertainty in the fractionation exponent (Figure 6), which in turn depends on the uncertainties in the $\mathrm{SEP}$ and solar wind isotope ratios.

If $\mathrm{C}$ is fully ionized, as we have assumed, then we find that $\mathrm{N}$ and $\mathrm{O}$ have $\sim 1$ electron attached and $\mathrm{Ne}$ through $\mathrm{S}$ have $\sim 2$. This He-like structure for $\mathrm{Ne}-\mathrm{S}$ was previously suggested (Reames et al. 1994) to explain the comparable enhancement of these elements relative to $\mathrm{CNO}$ and led to the suggestion that the source material is at a temperature of a few MK since the required charge states are present at those temperatures. Between $\mathrm{S}$ and $\mathrm{Ar}$, we find an abrupt increase in $Z-Q_{Z}$ by $\sim 2.5-3$ attached electrons followed by an essentially constant

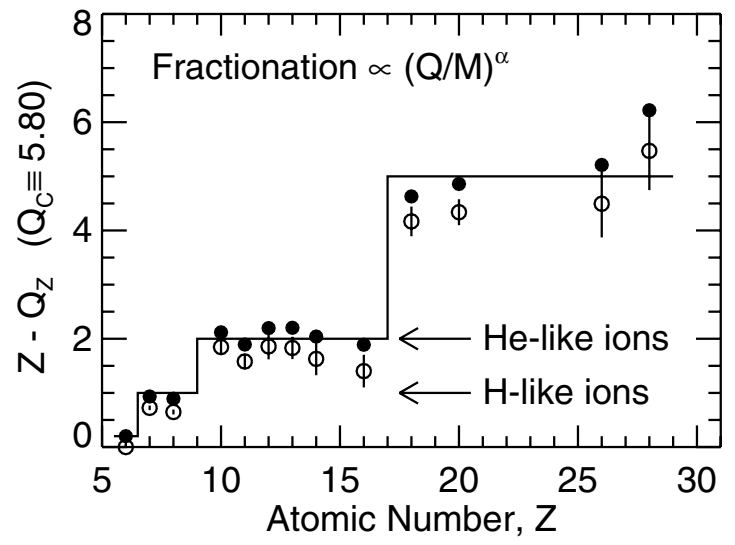

Figure 7. Mean ionic charge states (expressed in terms of the number of attached electrons, $Z-Q_{Z}$ ) inferred for a variety of elements in the 2002 August 20 event assuming that fractionation goes as a power law in $Q / M$. Open circles: values of $Q_{Z}$ obtained assuming that $Q_{\mathrm{C}}=6.0$. Filled circles: values obtained assuming that $Q_{\mathrm{C}}=5.8$ yield a more well-defined step structure with integral values of $Z-Q_{Z}$ for elements heavier than $\mathrm{C}$, as shown by the solid line. Error bars on the filled points are omitted for clarity.

value from Ar through Fe. All of the $Q_{Z}$ values scale proportional to the assumed value of $Q_{\mathrm{C}}$. If, for example, $Q_{\mathrm{C}}=5.8$, then the derived value of $Q_{\mathrm{Fe}}$ would be reduced by $\sim 0.7$ charge unit and the value of $Z-Q_{Z}$ correspondingly increased. Changing the assumed $Q_{\mathrm{C}}$ does not affect the conclusion that there are step increases in the number of attached electrons between $\mathrm{C}$ and $\mathrm{N}$, between $\mathrm{O}$ and $\mathrm{Ne}$, and between $\mathrm{S}$ and Ar. In fact, as illustrated by the filled points in Figure 7, assuming $Q_{\mathrm{C}}=$ 5.8 leads to a more well-defined step structure with one, two, and five electrons attached for $\mathrm{N}$ and $\mathrm{O}, \mathrm{Ne}-\mathrm{S}$, and $\mathrm{Ar}-\mathrm{Ni}$, respectively.

When an element in the source is present in several ionization states, the mean $Q$ after fractionation is given by an average of the form

$$
\left\langle Q_{Z}\right\rangle=\sum_{i} f_{i}\left[Q_{Z}^{i}\right]^{\alpha+1} / \sum_{i} f_{i}\left[Q_{Z}^{i}\right]^{\alpha}
$$

where $f_{i}$ is the fraction of element $Z$ in charge state $Q_{Z}^{i}$ in the source material. The large (negative) fractionation exponent causes deviation of the mean charge states in the fractionated material toward smaller values relative to the mean charge states present in the source. Similar considerations apply to the effects of having multiple isotopes of an element in the source (see Section 4.2).

\section{3. $Q_{F e}$ Estimate from Minimal Composition Data}

The 2002 August 20 event was unusual among impulsive events observed using $A C E$ /SIS during solar cycle 23 in that it had high enough intensity above $10 \mathrm{MeV}$ nucleon $^{-1}$ to allow measurements of the abundances of a large number of elements and isotopes, as discussed in Section 2. It is possible to obtain useful charge state information from the technique discussed above using only two abundance ratios, ${ }^{22} \mathrm{Ne} /{ }^{20} \mathrm{Ne}$ and $\mathrm{Fe} /$ $\mathrm{C}$. The isotope ratio provides the fractionation exponent and that exponent, in conjunction with the $\mathrm{Fe} / \mathrm{C}$ ratio, yields an estimate of $Q_{\mathrm{Fe}}$, which is generally the most useful ionic charge for characterizing the state of the material being fractionated. Written out explicitly this amounts to

$$
Q_{\mathrm{Fe}}=Q_{\mathrm{C}} \frac{M_{\mathrm{Fe}}}{M_{\mathrm{C}}} \exp \left[-\ln (22 / 20) \frac{\ln \mathcal{E}_{\mathrm{Fe} / \mathrm{C}}}{\ln \mathcal{E}_{22 / 20}}\right],
$$


with the approximate values $Q_{\mathrm{C}} \simeq 6, M_{\mathrm{C}} \simeq 12$, and $M_{\mathrm{Fe}} \simeq 56$. Here, $\mathcal{E}_{22 / 20}$ and $\mathcal{E}_{\mathrm{Fe} / \mathrm{C}}$ denote the enhancements of ${ }^{22} \mathrm{Ne} /{ }^{20} \mathrm{Ne}$ and $\mathrm{Fe} / \mathrm{C}$, respectively, in the SEPs relative to their values in the solar wind. Equation (4) provides a means for estimating $Q_{\mathrm{Fe}}$ that can be used even in the more-typical small impulsive events, provided that measurements of ${ }^{22} \mathrm{Ne} /{ }^{20} \mathrm{Ne}$ and $\mathrm{Fe} / \mathrm{C}$ are available. The relative uncertainty in the estimate of $Q_{\mathrm{Fe}}$ is then

$$
\begin{aligned}
\frac{\delta Q_{\mathrm{Fe}}}{Q_{\mathrm{Fe}}}= & \frac{\ln (22 / 20)}{\ln \mathcal{E}_{22 / 20}} \cdot\left[\left(\frac{\delta \mathcal{E}_{\mathrm{Fe} / \mathrm{C}}}{\mathcal{E}_{\mathrm{Fe} / \mathrm{C}}}\right)^{2}\right. \\
& \left.+\left(\frac{\ln \mathcal{E}_{\mathrm{Fe} / \mathrm{C}}}{\ln \mathcal{E}_{22 / 20}}\right)^{2}\left(\frac{\delta \mathcal{E}_{22 / 20}}{\mathcal{E}_{22 / 20}}\right)^{2}\right]^{1 / 2},
\end{aligned}
$$

with $\delta \mathcal{E}_{\mathrm{Fe} / \mathrm{C}}$ and $\delta \mathcal{E}_{22 / 20}$ denoting the uncertainties in the enhancement factors for $\mathrm{Fe} / \mathrm{C}$ and ${ }^{22} \mathrm{Ne} /{ }^{20} \mathrm{Ne}$. Applying Equations (4) and (5) to the 2002 August 20 event using the enhancement factors and uncertainties given in Tables 1 and 2 yields $Q_{\mathrm{Fe}} \simeq 21.7 \pm 0.6$, consistent with the value shown in Figure 7.

\section{INTERPRETATION OF THE INFERRED CHARGE STATES}

Direct measurements of charge states below $1 \mathrm{MeV}$ nucleon ${ }^{-1}$ (Klecker et al. 2007, and references therein) have shown that an increase of $Q_{\mathrm{Fe}}^{\text {obs }}$ by a few charge units between $\sim 0.2$ and $\sim 0.5 \mathrm{MeV}$ nucleon ${ }^{-1}$ occurs in the majority of impulsive SEP events. At energies $\lesssim 0.1 \mathrm{MeV}$ nucleon ${ }^{-1}, Q_{\mathrm{Fe}}^{\text {obs }}$ in most impulsive events is lower than in the $0.2-0.5 \mathrm{MeV}^{2}$ nucleon $^{-1}$ range (Klecker et al. 2005; DiFabio et al. 2008), with values similar to those found in the solar wind, although on rare occasions high $Q_{\mathrm{Fe}}^{\text {obs }}$ values $(\gtrsim 16)$ have been observed at these low energies (Guo et al. 2008). It is commonly thought that the $Q_{\mathrm{Fe}}^{\mathrm{obs}}$ value observed at the lowest energy is characteristic of the source plasma and that the increase of $Q_{\mathrm{Fe}}^{\text {obs }}$ at higher energies results from collisional stripping in a relatively dense medium (product of acceleration time times density exceeding a few $\times 10^{8} \mathrm{~s} \mathrm{~cm}^{-3}$ ) during acceleration (Klecker et al. 2006a; Kartavykh et al. 2008).

Measurements of $Q_{\mathrm{Fe}}^{\mathrm{obs}}$ from ACE/SEPICA, which provided much of the information about $Q_{\mathrm{Fe}}^{\text {obs }}$ trends below $1 \mathrm{MeV}$ nucleon $^{-1}$, were not available after 2000 . However, preliminary analysis of charge states at $1 \mathrm{AU}$ in the 2002 August 20 event using the geomagnetic cutoff technique with the SAMPEX/LICA instrument (J. Mazur 2008, private communication) yielded $Q_{\mathrm{Fe}}^{\mathrm{obs}}=18 \pm 1$ at energies of $0.25-0.5 \mathrm{MeV}$ nucleon $^{-1}$, increasing to $22.9 \pm 0.7$ at $0.5-1 \mathrm{MeV}$ nucleon $^{-1}$. In addition, it was found that $Q_{\mathrm{O}}^{\mathrm{obs}}=7.8$ \pm 0.2 in the higher-energy interval. These values may be subject to additional uncertainty, not included in the indicated errors, because a relatively large impulsive event on 2002 August 19 was probably contributing some of the particles used in these measurements. (For the higher velocity heavy ions measured with SIS, there is relatively little contribution from the 2002 August 19 event, as illustrated in Figure 1.)

Comparisons can be made between the value of $Q_{\mathrm{Fe}}$ that we derived assuming fractionation as a power law in $Q / M$ and distributions of $Q_{\mathrm{Fe}}^{\text {obs }}$ observed in various samples of solar material. Figure 8 compares the inferred Fe charge states with measured distributions of $Q_{\mathrm{Fe}}^{\text {obs }}$ in impulsive and gradual SEP events (upper and middle panels, respectively) and in various types of solar wind (lower panel). For both impulsive and gradual SEP events, distributions are shown at two different energies

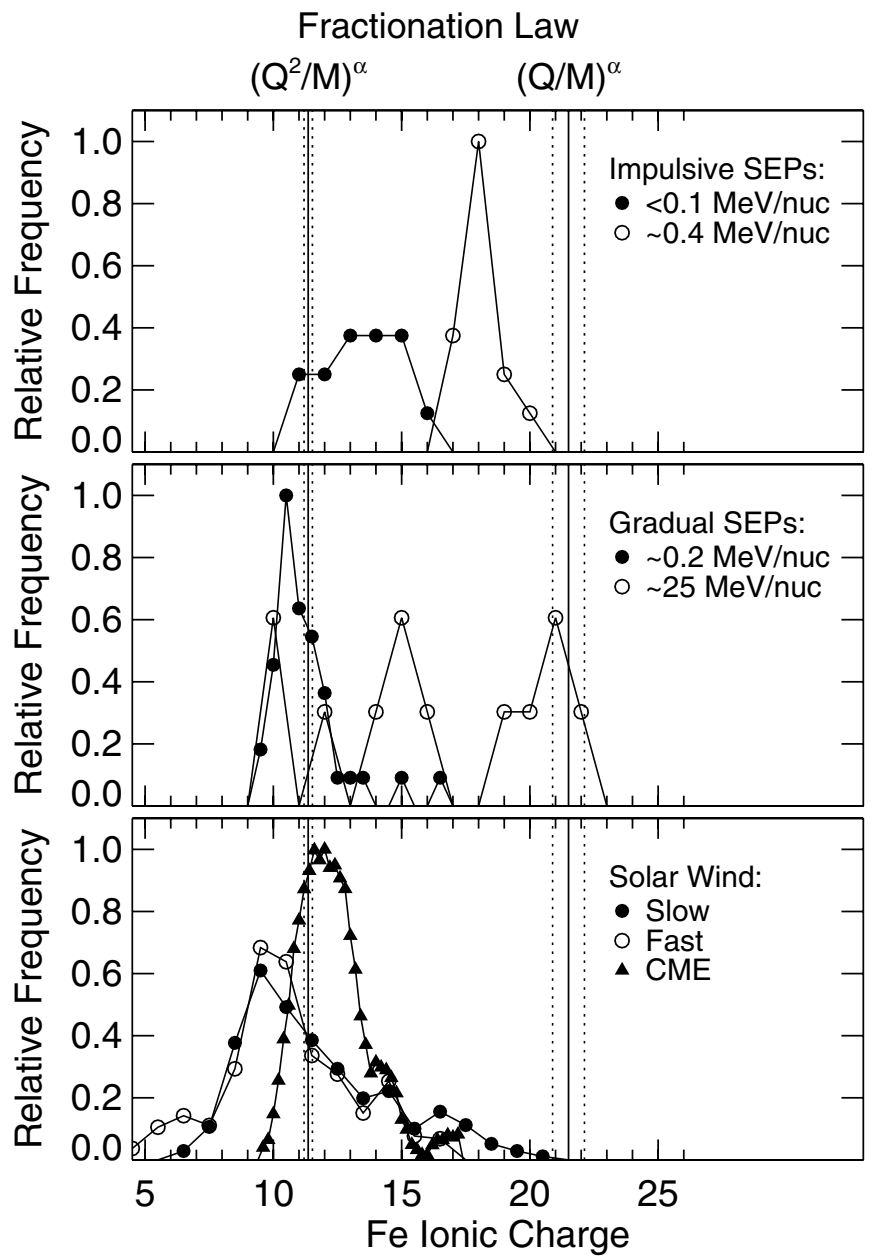

Figure 8. Comparison of inferred $Q_{\mathrm{Fe}}$ values in the 2002 August 20 event (solid vertical lines between dotted lines indicating the uncertainties) with measured distributions of $Q_{\mathrm{Fe}}^{\mathrm{obs}}$ in other samples of matter from the Sun. (The $\left(Q^{2} / M\right)^{\alpha}$ fractionation law is discussed in the Appendix.) Upper panel: impulsive SEP events at two different energies (DiFabio et al. 2008); middle panel: gradual SEP events at low energies (Klecker et al. 2007) and high energies (Labrador et al. 2003 and A. W. Labrador 2008, private communication); lower panel: fast and slow solar wind (Lepri et al. 2001) and solar wind in ICMEs (Lepri \& Zurbuchen 2004). The multiple peaks in the distribution of $Q_{\mathrm{Fe}}^{\mathrm{obs}}$ values in the high-energy gradual SEP distribution are caused by statistical fluctuations associated with the small number of events studied.

and it is clear that the higher-energy sample is characterized by a higher average value of $Q_{\mathrm{Fe}}^{\text {obs }}$ (see review of Klecker et al. 2006a). For both types of events, this has been interpreted as being the result of collisional stripping during acceleration (see Barghouty \& Mewaldt 1999, 2000; Kartavykh et al. 2005, and references therein), although other explanations have also been proposed for the energy dependence in gradual events (Tylka et al. 2001). Our value of $Q_{\mathrm{Fe}}$ (Figure 8, right-hand vertical band) is comparable to the highest values found in gradual events at similar energies. It exceeds the $Q_{\mathrm{Fe}}^{\text {obs }}$ values shown for the higher-energy $\left(\sim 0.4 \mathrm{MeV}\right.$ nucleon $\left.{ }^{-1}\right)$ impulsive-event population in Figure 8, possibly because the data we are using from $A C E / S I S$ are at a still higher energy. Furthermore, as noted above, there are indications from the SAMPEX/LICA data that near $1 \mathrm{MeV}$ nucleon $^{-1}$ the $\mathrm{Fe}$ is close to fully stripped in the 2002 August 20 event. In Section 4.3, we show that the inferred charge states in the 2002 August 20 event are very similar to those that have been derived for several events that exhibited a mix of gradual and impulsive SEP characteristics. 
It should be kept in mind that the distributions shown in the upper and middle panels of Figure 8 refer to charge states of $\mathrm{Fe}$ ions arriving at $1 \mathrm{AU}$. On the other hand, the inferred charge states that we derive should be indicative of the ionization state at the time and place where the elemental and isotopic fractionation occurred. At the energies $\gtrsim 0.1 \mathrm{MeV}$ nucleon $^{-1}$ being considered here electron stripping is significantly more probable than electron attachment (Kocharov et al. 2000), so during acceleration and transport in a medium where collisions occur charge states increase with time. Thus, we interpret the fact that our inferred value of $Q_{\mathrm{Fe}}$ during the fractionation is higher than typical values of $Q_{\mathrm{Fe}}^{\mathrm{obs}}$ measured in situ at $\sim 0.5 \mathrm{MeV}$ nucleon $^{-1}$ to mean that the fractionation occurred after the acceleration and stripping that produces the $\mathrm{Fe}$ charge states measured below $0.5 \mathrm{MeV}$ nucleon ${ }^{-1}$. This argument assumes, of course, that events such as 2002 August 20 that are detected above $10 \mathrm{MeV}$ nucleon $^{-1}$ have characteristics below $1 \mathrm{MeV}$ nucleon $^{-1}$ similar to those of the more common population of impulsive events studied below $1 \mathrm{MeV}$ nucleon $^{-1}$ (Klecker et al. 2007; DiFabio et al. 2008, and references therein). This conclusion is weakened if acceleration, stripping, and fractionation cannot be adequately modeled as discrete, sequential processes as we have assumed.

Luhn et al. (1987) reported in situ measurements of charge states at energies $\sim 0.3-3 \mathrm{MeV}$ nucleon $^{-1}$ in a number of impulsive events. They found $Q_{\mathrm{Si}}^{\mathrm{obs}}>11.7$ and $Q_{\mathrm{Fe}}^{\mathrm{obs}}=20.5 \pm$ 1.2 , consistent with our inferred charge states. These authors took the $\mathrm{Si}$ result as an indication that $\mathrm{Si}$ is fully ionized and argued that it could not have experienced fractionation relative to other $M / Z=2$ nuclides, assuming that the fractionation is a function of $Q / M$. However, when the $Q / M$ dependence is as strong as that which we find in the 2002 August 20 event, differences of 1-2 charge units from being fully stripped are sufficient to produce significant relative enhancements.

DiFabio et al. (2008) argued against a scenario in which fractionation occurs after most of the stripping, largely because no mechanism capable of producing this fractionation has been identified. Identification of the physical process responsible for the fractionation is indeed one of the key open questions in interpreting the observed elemental and isotopic abundances.

\subsection{Comparison with Thermal Charge States}

By comparing with the calculated charge state distributions of Mazzotta et al. (1998), we have investigated whether the inferred charge states shown in Figure 7 could arise from a plasma in thermal equilibrium. Since the technique we are using yields only ratios between charge states of different elements, one can scale all the inferred $Q_{Z}$ values by a common factor. For each temperature between $0.5 \mathrm{MK}$ and $100 \mathrm{MK}$ in the Mazzotta et al. (1998) tabulation, we found the scaling factor that gives the best agreement (smallest $\chi_{v}^{2}$ ) between the inferred and calculated mean charges. Only scaling factors that met the condition $Q_{Z}-\sigma_{Q_{Z}} \leqslant Z$ for all the elements were allowed, where $\sigma_{Q_{Z}}$ denotes the uncertainty in the derived value of $Q_{Z}$. The resulting $\chi_{v}^{2}$ versus temperature curve has a broad minimum around $\sim 4 \mathrm{MK}$. Figure 9 compares our $Q_{Z}$ values using the best scaling (points) with the calculated values for this temperature (heavy solid curve) and for a number of other temperatures (dotted curves). Note that since the scale factor applied to the points is temperature dependent, only an approximate comparison between the points and the dotted curves is possible from this plot. Furthermore, in calculating $\chi_{v}^{2}$ values only the uncertainties in the inferred charge states are taken into account;

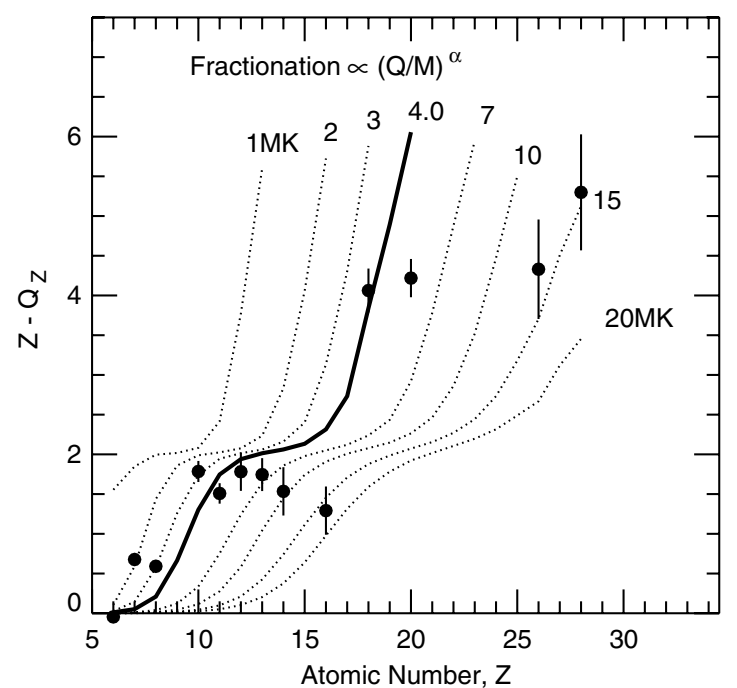

Figure 9. Comparison of charge states (plotted in the form $Z-Q_{Z}$ ) inferred assuming fractionation going as $(Q / M)^{\alpha}$ with calculated equilibrium charge states as a function of temperature (Mazzotta et al. 1998). The heavy curve corresponds to the best-fit temperature of $4.0 \mathrm{MK}$. Over the range $6 \leqslant Z \leqslant 28$, the inferred charge states are not consistent with a single isothermal source. See the text for details.

no estimate of the uncertainty in the calculated thermal charge states is included. The resulting minimum $\chi_{v}^{2}$ value is large, $\sim 44$, consistent with the overall poor quality of the fit shown in the figure. There is a clear tendency for the heavier elements, and particularly $\mathrm{Fe}$ and $\mathrm{Ni}$, to be more ionized than expected in a 4 MK plasma. If only the $\mathrm{Fe}$ and $\mathrm{Ni}$ charge states, which are derived from the abundance enhancements of these elements relative to that of $\mathrm{C}$, are used for inferring a temperature, a value $\sim 15$ MK results. Overall, we find no strong evidence that the inferred charge states arise directly from an isothermal plasma.

Reames et al. (1994) studied the relative enhancements of the elements from $\mathrm{C}$ through $\mathrm{Si}$. Assuming that the fractionation depends on $Q / M$, they inferred source temperatures in the range 2.5-5.0 MK. Comparing the enhancements of $\mathrm{Fe}$ and $\mathrm{NeMgSi}$ and allowing the possibility that the Fe source temperature need not be the same as that for $\mathrm{NeMgSi}$, these authors argued against temperatures above $10-15 \mathrm{MK}$ for Fe because for $Q_{\mathrm{Fe}} \geqslant 21 \mathrm{Fe}$ would have a similar $Q / M$ value as the He-like ions $\mathrm{NeMgSi}$ and would no longer be selectively enhanced. The results we obtain assuming fractionation as a power law in $Q / M$ are, for the most part, consistent with those of Reames et al. (1994). The main difference is that the $(Q / M)^{-15}$ dependence that we derive from the isotope fractionation could produce the observed enhancement of Fe relative to $\mathrm{Mg}^{+10}$ of $4.3(15 / 3.5$, Table 1) with a difference in $Q / M$ of only $10 \%$, corresponding to $\mathrm{Fe}^{+23.3}$.

Values of $Q_{\mathrm{Fe}}^{\text {obs }} \gtrsim 20$ obtained from the SAMPEX/LICA measurements at 1 AU for the 2002 August 20 event (J. Mazur 2008, private communication) are similar to the $Q_{\mathrm{Fe}}$ values from our analysis of the elemental and isotopic fractionation, assuming that this process has the form of a power law in $Q / M$. This is consistent with a scenario in which the fractionation takes place after acceleration to above $0.5 \mathrm{MeV}$ nucleon $^{-1}$ and after most of the stripping has occurred. In this case, one would not expect our inferred ionic charge states to be consistent with equilibrium values in an isothermal plasma.

\subsection{Origin of the Elemental Enhancements}

In the case of fractionation $\propto(Q / M)^{\alpha}$, the pattern seen in the plot of elemental abundance enhancements versus $Z$ (Figure 4) 


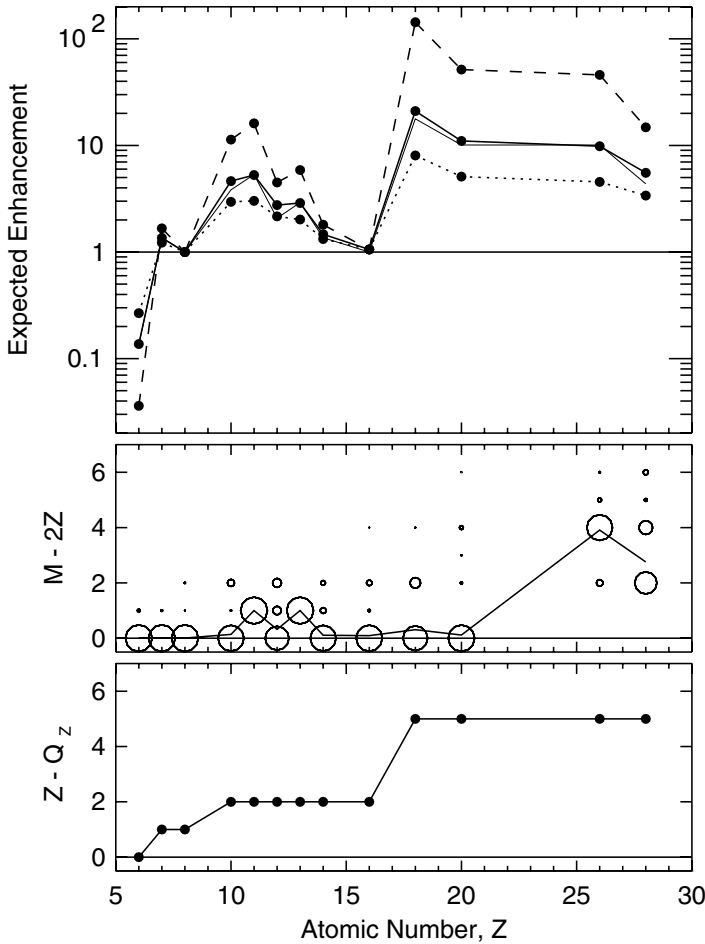

Figure 10. Elemental abundance enhancements (upper panel) normalized to oxygen calculated based on a simple $Z$ dependence of the number of attached electrons (lower panel) and known masses (middle panel) and assuming fractionation $\propto(Q / M)^{\alpha}$. The areas of the circles in the middle panel are proportional to the isotopic abundances in solar system material and the curve indicates the mean masses for the elements. The solid curves in the upper panel are for a fractionation exponent $\alpha=-15.0$ as found in the 2002 August 20 event. Two additional cases of more extreme ( $\alpha=-25$, dashed line) and less extreme ( $\alpha=-10$, dotted line) fractionation are also shown. The thin solid line was calculated using only the mass of the most abundant isotope to derive the enhancement, as was done in analyzing the data from the 2002 August 20 event. The other curves took all of the isotopes into account.

can be interpreted as arising from a combination of the steps in the number of attached electrons as a function of $Z$ (Figure 7) and the mass numbers of the nuclides. To illustrate this, we have calculated the elemental abundance enhancements that would be expected from $Q / M$ fractionation with an exponent of $\alpha=-15$ in combination with charge states corresponding to $\mathrm{C}$ being fully stripped, $\mathrm{N}$ and $\mathrm{O}$ having one attached electron, Ne through $\mathrm{S}$ having two, and Ar through Ni having five. This pattern, shown in the plot of $Z-Q_{Z}$ versus $Z$ in the lower panel of Figure 10, approximates the pattern derived from the composition data in the 2002 August 20 event.

The middle panel shows the masses of each element's stable isotopes expressed in terms of the neutron excess, $M-2 Z$. The points are plotted with areas proportional to the relative isotope fractions in solar system material (Lodders 2003). The solid curve shows the mean masses of the elements and indicates that only for a few elements does the mean mass differ appreciably from the mass of the most abundant isotope. Expected enhancements were calculated separately for each stable isotope of an element and the results averaged using the solar system abundances of the isotopes (Lodders 2003) as weights.

The heavy solid curve in the upper panel of Figure 10 shows the pattern of abundance enhancements obtained by combining values of $Q$ from the lower panel and $M$ from the middle panel with the $(Q / M)^{\alpha}$ fractionation law for $\alpha=-15$. Due to the negative exponent, an increase in $Q$ causes a decrease in the abundance enhancement whereas an increase in $M$ causes an increase in the enhancement. The shape of the curve, which is quite similar to the plot of measured elemental enhancements shown in Figure 4, illustrates how the structure in the $\mathcal{E}_{Z}$ versus $Z$ curve could originate.

1. The elements $\mathrm{C}$ and $\mathrm{N}$ both have $Q \simeq 6$, but since $\mathrm{N}$ is 2 amu more massive it experiences a larger enhancement.

2. Going from $\mathrm{N}$ to $\mathrm{O}, Q$ increases by $\sim 1$ unit (both elements have $\sim 1$ electron attached), so $Q$ increases by a factor of $\sim 7 / 6$ while the mass increases by a smaller factor, $16 / 14$, causing $Q / M$ to increase and the fractionation to decrease.

3. The addition of a second electron going from $\mathrm{O}$ to $\mathrm{Ne}$ causes $Q$ to increase by a factor $\sim 8 / 7$, but since this factor is significantly smaller than the mass increase $(20 / 16)$, a sharp increase in the enhancement results.

4. Progressing through the elements $\mathrm{Ne}, \mathrm{Mg}, \mathrm{Si}$, and $\mathrm{S}$, where all of the ions have a He-like structure with two attached electrons, both the charge and the mass increase uniformly with $Z$, but the fractional rate of charge increase is greater (because of the two electrons), so the enhancement decreases smoothly.

5. An exception to this smooth trend occurs for $\mathrm{Al}(Z=13)$ because this odd $Z$ nucleus has a mass number $M=2 Z+1$ rather than the $2 \mathrm{Z}$ of $\mathrm{Ne}, \mathrm{Mg}, \mathrm{Si}$, and $\mathrm{S}$. The result is a small peak at $\mathrm{Al}$.

6. The element $\mathrm{Na}(Z=11)$, which also has $M=2 Z+1$, shows a small peak in the expected enhancement curve. It is less pronounced than that for Al. In the enhancements derived for the 2002 August 20 event, the Na enhancement is, within errors, equal to that for $\mathrm{Ne}$.

7. The addition of three more electrons between $\mathrm{S}$ and $\mathrm{Ar}$ results in the value of $Q$ decreasing slightly (factor of 13/ 14) while the mass increases by a factor $36 / 32$, producing the abrupt increase in the enhancement between these two elements.

8. Between Ar and Fe the charge increase by a factor $\sim 1.6$ is approximately matched by the mass increase because the mass distributions of the elements are becoming neutron rich. This causes the enhancement plateau for the heavier elements.

In deriving the charge states for the 2002 August 20 event shown in the upper panel of Figure 7, we assumed a mass for each element equal to that of the most abundant isotope. To investigate how large an error is caused by this approximation, we have plotted as the thin solid line in Figure 10 the elemental enhancement pattern that would be obtained using only the mass of the most abundant isotope. The difference between the heavy and light solid curves is typically smaller than the uncertainties in the elemental enhancements shown in Figure 4.

In principle, one should also take into account the fact that the elements will actually have a distribution of charge states rather than the single, well-defined value we have assumed. However, we have argued above (Section 4.1) that the $Z$-dependent temperatures inferred from the derived $Q$ values (Figure 9) are probably not indicative of the state of a source in thermal equilibrium (which would allow the distribution of $Q_{Z}$ to be calculated). It is more likely that the inferred values of $Q_{Z}$ have been significantly affected by non-thermal processes such as collisional stripping. Thus, at present we do not have enough information to allow us to take charge state distributions meaningfully into account. Furthermore, the inferred pattern of integral steps in the number of attached electrons as a function of 
$Z$ (Figure 7) seems unlikely unless the charge state distributions are dominated by single ionization states.

As shown by Wiedenbeck et al. (2008, 2009), the same qualitative pattern of elemental abundance enhancements is present in a sizeable fraction of the impulsive SEP events large enough for $A C E$ /SIS to obtain good elemental composition measurements above $10 \mathrm{MeV}$ nucleon $^{-1}$. However, the enhancement of the ${ }^{22} \mathrm{Ne} /{ }^{20} \mathrm{Ne}$ ratio has significant variation from event to event, which would be indicative of differences in the fractionation exponent $\alpha$. To check how the elemental abundance pattern would vary with changes in $\alpha$ (while leaving the $Q_{Z}$ values unchanged), we have calculated expected enhancements assuming $\alpha=-10$ and $\alpha=-25$, which covers most of the cases that have been observed. The results are shown in the upper panel of Figure 10 where the dashed line corresponds to $\alpha=-25$ and the dotted line to $\alpha=-10$. Although an elemental abundance ratio such as $\mathrm{Fe} / \mathrm{C}$ can change by a factor $>20$ between these two values of $\alpha$, the peaks, valleys, and plateaus in the enhancement pattern remain reasonably clear. Thus, the persistence of this same pattern over events with a variety of ${ }^{22} \mathrm{Ne} /{ }^{20} \mathrm{Ne}$ ratios suggests that the pattern of charge states does not differ significantly from event to event, although the strength of the fractionation does. In Section 4.3, we compare the fractionation pattern in the 2002 August 20 event with patterns that have been reported from several previous heavy-ion composition studies.

Another striking feature seen in the upper panel of Figure 10 is the constancy of the S/O ratio over a wide range of fractionation exponents, which is clearly the result of the two elements having the same $Q / M$ value $(7 / 16$ and $14 / 32$, neglecting the rare isotopes). In the sample of impulsive events for which heavy element abundances from $A C E /$ SIS were reported by Wiedenbeck et al. (2008), S/O enhancements range between $\sim 1$ (as in the 2002 August 20 event) and $\sim 3$, while the compilations of Reames (1995a) and Mason et al. (2004) imply average values $\sim 2.35$. This would indicate that $Q / M$ for S tends to be slightly less than the value for $\mathrm{O}$ in most events. Other pairs of elements with nearly identical $Q / M$ values could also occur, but would not be as evident as the case of $\mathrm{S}$ and $\mathrm{O}$ since we have used $\mathrm{O}$ for normalizing the abundances. Assuming that ${ }^{22} \mathrm{Ne} /{ }^{20} \mathrm{Ne}$ has a large event-to-event variation, as appears to be the case (Wiedenbeck et al. 2008, 2009), it should be possible to obtain useful information about the charge states of some elements simply by looking for element pairs whose abundance ratio does not vary significantly over the set of events (see Reames et al. 1994).

The ability to account for the pattern of elemental abundance enhancements in terms of $(Q / M)^{\alpha}$ fractionation of a population of source material in which the charge states are characterized by several step increases in the number of attached electrons as a function of $Z$ does not, of course, provide a full explanation of the impulsive SEP event composition. The key remaining questions are (1) how is the step-like pattern of charge states produced and (2) what is the physical mechanism causing the $(Q / M)^{\alpha}$ fractionation.

\subsection{Comparison with Other Fractionation Studies}

In the discussion of the charge states and associated source temperatures derived from our analysis assuming $Q / M$ fractionation (Sections 3.2 and 4.1), we noted that Reames et al. (1994) came to generally the same conclusions from their examination of the pattern of elemental enhancements in impulsive SEP events. While encouraging, this agreement does not provide independent evidence that $Q / M$ is the physical variable controlling the fractionation. The arguments used by Reames et al. (1994) were predicated on the assumption that ions with the same value of $Q / M$ experience the same enhancement.

As mentioned above, Cohen et al. (1999a) originally applied the technique we are using for inferring charge states assuming fractionation as a power law in $Q / M$ in a study of several relatively large SEP events that occurred in 1997 and 1998. Four of the nine events studied had measurable enhancements of ${ }^{3} \mathrm{He}$, but with ${ }^{3} \mathrm{He} /{ }^{4} \mathrm{He}<0.1$. The four events were generally similar to the 2002 August 20 event in terms peak heavy-ion intensity averaged over the $12-60 \mathrm{MeV}$ nucleon ${ }^{-1}$ band with, for example, the oxygen intensity ranging from $\sim 1.2 \times 10^{-4}$ to $\sim 1.4 \times 10^{-3}\left(\mathrm{~cm}^{2} \text { sr s MeV nucleon }{ }^{-1}\right)^{-1}$ as compared with $\sim 3.0 \times 10^{-4}$ for 2002 August 20 (see Figure 1). Cohen et al. (1999a) showed that the pattern of elemental abundances in these events agrees significantly better with typical impulsiveevent abundances than with typical gradual-event abundances (Reames 1995a). In addition, the inferred values of $Q_{\mathrm{Fe}}$ were $>19$, in the range considered typical of impulsive SEP events and significantly greater than those thought to be characteristic of gradual events (Reames 1995b). In the most intense of these events (1997 November 6), measurements using the geomagnetic cutoff technique (Mazur et al. 1999) showed $Q_{\mathrm{Fe}}^{\mathrm{obs}}$ increasing from $\sim+11$ below $1 \mathrm{MeV}$ nucleon ${ }^{-1}$ to $>+15$ above $35 \mathrm{MeV}$ nucleon $^{-1}$. Although these events might be classified as impulsive in terms of the characteristics noted above, they also had some features that would suggest they are actually gradual events involving shock acceleration. These included high proton intensities above $10 \mathrm{MeV}$ and, at least in some cases, temporal association with a broad, fast CME.

Using the elemental composition data from Cohen et al. (1999a) and the measurements of the ${ }^{22} \mathrm{Ne} /{ }^{20} \mathrm{Ne}$ isotope ratio from Leske et al. (1999), together with the solar wind abundances listed in Tables 1 and 2, we have calculated the number of attached electrons, $Z-Q_{Z}$, assuming fractionation $\propto(Q / M)^{\alpha}$. The calculation for $Q / M$ fractionation is essentially that performed by Cohen et al. (1999a), but using solar wind abundances for normalization for consistency with the present work. As shown in Figure 11, the inferred charge states in the Cohen et al. (1999a) events follow a very similar pattern to those in the 2002 August 20 event, with the same steps in the number of attached electrons between $\mathrm{C}$ and $\mathrm{N}, \mathrm{O}$ and $\mathrm{Ne}$, and $\mathrm{S}$ and $\mathrm{Ar}$, as well as the plateau between $\mathrm{Ar}$ and $\mathrm{Fe}$. In three of the Cohen et al. (1999a) events, the number of attached electrons for $\mathrm{Ca}$ through $\mathrm{Ni}$ is greater than in the 2002 August 20 event by $\sim 1-3$, while in the 1998 May 6 event the charge states of $\mathrm{Ar}, \mathrm{Ca}$, and $\mathrm{Fe}$ are nearly the same as in the 2002 August 20 event (Cohen et al. (1999a) did not report a value for $Q_{\mathrm{Ni}}$ in the 1998 May 6 event). It may be significant that of the four events from the Cohen et al. (1999a) study, the 1998 May 6 event had the highest value of ${ }^{3} \mathrm{He} /{ }^{4} \mathrm{He}, \sim 0.04$, as compared with values between 0.005 and 0.01 in the other three events.

It should be noted that the events in the Cohen et al. (1999a) study have significantly different elemental composition than the 2002 August 20 event. For example, the $\mathrm{Fe} / \mathrm{C}$ ratios range between 2.0 and 2.8 while for the 2002 August 20 event that value is 9.3 (Table 1). Similarly, the fractionation exponents derived from the ${ }^{22} \mathrm{Ne} /{ }^{20} \mathrm{Ne}$ ratios of Leske et al. (1999) are $\sim-7$ for three of the events and $\sim-11$ for the 1998 May 6 event, as compared with -15 for 2002 August 20. In spite of the sizeable differences in elemental abundances and fractionation exponents, the inferred charge states calculated by combining these quantities yield rather consistent values. Based on the 


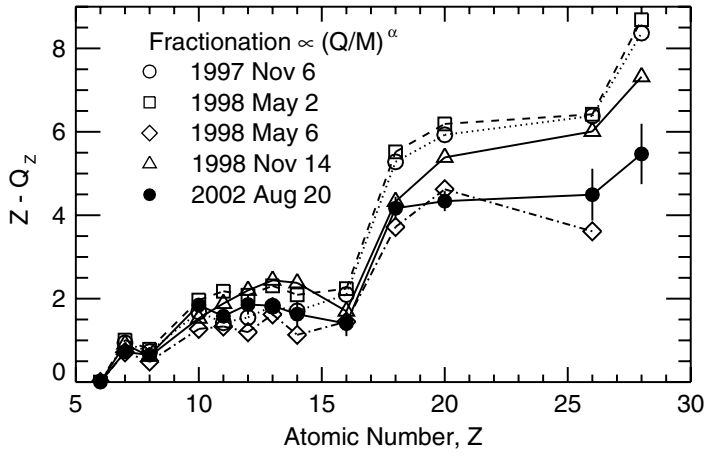

Figure 11. Comparison of inferred charge states in the 2002 August 20 impulsive SEP event with those derived for four SEP events studied by Cohen et al. (1999a). The pattern of charge states is similar in all of these events. In all cases, $Q_{\mathrm{C}}=$ 6.0 has been assumed for normalization.

inferred charge states and on the isotopic fractionation going as a steep power law in the mass ratio, we would suggest that all of these may be examples of the same type of event. The significance of these similarities in light of the gradual-event characteristics of the events in the Cohen et al. (1999a) study remains to be understood.

The composition in typical impulsive SEP events below $1 \mathrm{MeV}$ nucleon $^{-1}$ was measured by Mason et al. (2004) using $A C E$ /ULEIS and has been shown in Figure 4 (diamonds). This study included 20 events with ${ }^{3} \mathrm{He} /{ }^{4} \mathrm{He}$ ratios ranging between 0.002 and 0.4. It was found that the average enhancements of these impulsive-event abundances relative to abundances in gradual SEP events (Reames 1995a), which have at times been used as a proxy for coronal abundances, are generally organized as a decreasing function of $Q / M$ if one assumes values of $Q$ expected in a 3.2 MK plasma. A very similar result was reported by Reames \& Ng (2004). Mason et al. (2004) fit this dependence using a power law in $Q / M$ and found an exponent of -3.26 , although with some significant deviations. When we attempt to account for the charge states in the 2002 August 20 event in terms of a thermal source (see Section 4.1 and Figure 9), we find a somewhat higher temperature, $\sim 4 \mathrm{MK}$ for elements below $\mathrm{Fe}$, also with significant deviations. More striking, perhaps, is the large difference in fractionation exponents used in accounting for rather similar elemental enhancement patterns: $\alpha=-15$ in the present study and -3.26 in the Mason et al. (2004) work. We also note that Dwyer et al. (2001) found that the ${ }^{22} \mathrm{Ne} /{ }^{20} \mathrm{Ne}$ isotope ratio tends to be enhanced by factors in the range $\sim 5-10$ when ${ }^{3} \mathrm{He} /{ }^{4} \mathrm{He}$ is significantly greater than 0.1 , and $\sim 2-3$ for ${ }^{3} \mathrm{He} /{ }^{4} \mathrm{He} \simeq 0.1$. However, a fractionation exponent of -3.26 would produce an ${ }^{22} \mathrm{Ne} /{ }^{20} \mathrm{Ne}$ enhancement of only $\sim 1.4$. Given the wide range of ${ }^{22} \mathrm{Ne} /{ }^{20} \mathrm{Ne}$ values and implied fractionation exponents that are found both below $1 \mathrm{MeV}$ nucleon ${ }^{-1}$ (Dwyer et al. 2001) and above $10 \mathrm{MeV}$ nucleon ${ }^{-1}$ (Wiedenbeck et al. 2003, 2009), it could be important to analyze fractionation in individual impulsive events and not only in averages over many events.

Although the enhancement pattern seen in Figure 4 is typical of impulsive events, Mason et al. (2002b) reported three examples of impulsive SEP events observed with $A C E /$ ULEIS below $0.5 \mathrm{MeV}$ nucleon ${ }^{-1}$ that had unusual heavy element fractionation patterns. These events, all of which had ${ }^{3} \mathrm{He} /{ }^{4} \mathrm{He}>1$, had enhancements of $\mathrm{N}$ relative to $\mathrm{C}$ and to $\mathrm{O}$ that were even larger than we find in the 2002 August 20 event (Figure 4). This large $\mathrm{N}$ enhancement was accompanied by enhancements of $\mathrm{Si}, \mathrm{S}$, and $\mathrm{Ca}$ that significantly exceeded those of $\mathrm{Mg}$ and of

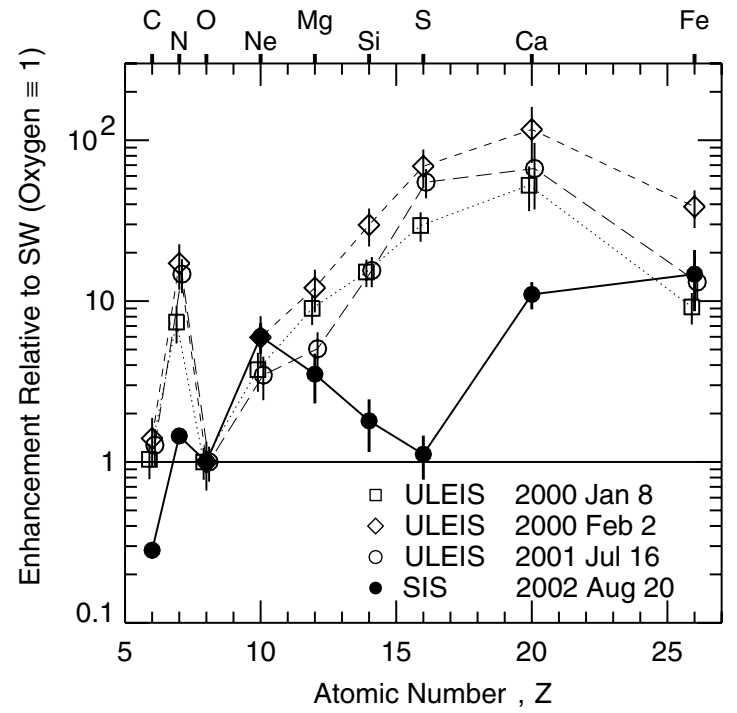

Figure 12. Comparison of elemental abundance enhancements measured by $A C E$ /SIS in the 2002 August 20 event with composition observed by $A C E /$ ULEIS in three events found to have a non-monotonic dependence of the enhancements on $Z$ (Mason et al. 2002a). Only those elements that were reported in the Mason et al. (2002a) study are shown. The same solar wind abundances were used for normalizing all four SEP data sets. For clarity, the points for the ULEIS events have been plotted displaced slightly from one another in $Z$.

Fe. In Figure 12, we compare the abundance enhancements reported by Mason et al. (2002a) in these three events with those obtained for the 2002 August 20 event in the present study, using the same set of solar wind abundances (Table 1) as the normalization. While both the 2002 August 20 event and the ULEIS events exhibit distinctly non-monotonic dependence of elemental abundance enhancements as a function of $Z$, the enhancement variations are more extreme in the ULEIS events and the patterns are sufficiently different so that it is not clear that these events are reflecting the same fractionation mechanism. It is striking that all three ULEIS events have the same qualitative pattern of abundance enhancements (which is distinctly different from the typical pattern found in impulsive events at ULEIS energies, shown by the diamonds in Figure 4) and that the pattern found in the 2002 August 20 event is also seen in most of the impulsive events that have been measured with SIS (Wiedenbeck et al. 2008, 2009).

It should be kept in mind, however, that the energies for the SIS and the ULEIS composition measurements differ by more than an order of magnitude. Given the energy dependence of $Q_{\mathrm{Fe}}^{\mathrm{obs}}$ that is normally observed below $0.5 \mathrm{MeV}^{\text {nucleon }}{ }^{-1}$ (Klecker et al. 2007), it is not inconceivable that a $Q$-dependent fractionation mechanism acting during or after the time of the acceleration and stripping could cause significantly different enhancement patterns at the two energies. None of the ULEIS events reported by Mason et al. (2002a) were detectable at SIS energies, and ULEIS did not measure the 2002 August 20 event. Thus, at present we lack direct evidence for a strong variation of enhancement patterns with energy in individual events.

\section{DISCUSSION}

\subsection{Solar Flare Acceleration Models}

Various models for fractionation in impulsive SEP events have been proposed based on resonant heating and/or resonant acceleration by particle interactions with plasma waves (Petrosian 2008; Petrosian et al. 2009; Zhang et al. 2005, and references 
therein). These interactions depend on $Q / M$ since ions with different $Q / M$ ratios resonate with different parts of the plasma wave spectrum. The resulting composition and spectra can also be influenced by particle diffusion out of the acceleration region and energy loss by collisions. These processes potentially introduce additional dependences on $Q / M$ due to the rigidity dependence of the diffusion coefficient (Möbius et al. 1980) and on $Q^{2} / M$ through the Coulomb energy loss rate (Mullan \& Levine 1981; Mullan 1983; Liu et al. 2006; Petrosian 2008). Although various power-law dependences on $Q$ and $M$ are expected from these theories, there is presently no consensus on the specific form of these dependences.

In a recent study, Drake et al. (2009) suggested a mechanism that could lead to a fractionation law going as a power law in $Q / M$. These authors noted that reconnection in solar flares normally involves a significant guide field perpendicular to the reconnecting component fields. Ion cyclotron periods depend on the strength of the guide field and on the $Q / M$ ratios. As ions are swept into the exhaust region where the plasma is flowing away from the magnetic X-line with approximately the Alfvén velocity, ions with cyclotron periods longer than the transit time across the boundary layer adjacent to the exhaust region can abruptly encounter this bulk plasma flow with its magnetic field. These particles then behave like classic pickup ions in the flow. Subsequent scattering of the ions converts the energy of the systematic motion relative to the plasma into random thermal motion, thus heating this population of ions. On the other hand, ions with cyclotron periods small compared to the time to be swept across the boundary layer behave adiabatically and are swept along with the exhaust flow without significant heating. As a result, there is a threshold for heating such that $Q / M$ must be less than some value that is proportional to the strength of the reconnecting field. This threshold is independent of the strength of the guide field because the dependences of the gyrofrequency and the boundary layer thickness on this quantity cancel (Drake et al. 2009).

Drake et al. (2009) noted that during reconnection current sheets fragment into multiple islands with a distribution of sizes in which the magnetic field strength, and thus the heating threshold, should be proportional to the size of the island. With the added assumption that the distribution of island sizes has the form of a power law, they find that the rate of production of suprathermal ions should be a power law in $Q / M$ that is three units shallower than the power law describing the island size distribution (Drake et al. 2009). The suprathermal ions produced by this mechanism would then have to undergo a second stage of acceleration that does not significantly alter the composition in order to produce the SEP population observed at $\mathrm{MeV}$ energies. A first-order Fermi process in the contracting magnetic islands may provide a mechanism for this second stage of acceleration, analogous to the mechanism proposed for electron acceleration by Drake et al. (2006).

Explanation of the fractionation observed in the 2002 August 20 event in terms of the mechanism proposed by Drake and collaborators would require an extremely steep distribution of island sizes, $w$, going as $\sim w^{-18}$, assuming the value of the fractionation exponent $\alpha=-15$ that we obtain from the isotope data (Section 2.3). In addition, the source material would need to have relatively high charge states (Figure 7). The temperatures $\gtrsim 4 \mathrm{MK}$ inferred assuming that charge states arise from a plasma in thermal equilibrium (Figure 9) are in the range found in flaring loops (Feldman 1996), but distinctly hotter than found in ambient coronal material, suggesting that the flare does not just fractionate material based on preexisting charge states, but also plays a role in producing the charge states. In view of this combination of requirements, the scenario put forward by Drake and collaborators does not appear to offer a likely explanation for the fractionation of the SEPs observed at high energies in events such as that of 2002 August 20.

\subsection{Plausibility of the Fractionation Law}

The dependence of the fractionation on $Q / M$ is reasonable from a physical point of view, as discussed above. In addition, the $Q / M$ power-law form has been suggested based on observations (Slocum et al. 2003; Mason et al. 2004; Reames $\& \mathrm{Ng}$ 2004). However, the large values of the power-law exponent, which was calculated to be $\alpha=-15$ in the 2002 August 20 event and even larger in some other impulsive events (Wiedenbeck et al. 2009), lead one to question whether there is a realistic physical mechanism that would give rise to such extreme power laws. It is, of course, conceivable that the power laws we are using to represent the fractionation are just approximations to some other fractionation law with a strong dependence on $Q / M$.

The strong dependence of the fractionation on $M$ is based directly on the data (Figure 6); a 10\% change in mass produces an enhancement $\sim 4$ in an isotope ratio such as ${ }^{22} \mathrm{Ne} /{ }^{20} \mathrm{Ne}$. When we assume that the mass dependence arises from a fractionation law depending on $Q / M$ and apply the powerlaw form to derive charge states, we are extrapolating the power law. The ratio of $Q / M$ values between $\mathrm{Ni}$ and $\mathrm{C}$ (Figure 7) is $\sim 1.3$. It is legitimate to ask whether extrapolation of the range of the power law by this amount (factor $\sim 3$ in the logarithm of the parameter controlling the fractionation) still provides an accurate representation of relative enhancement factors when values of $Q / M$ are significantly beyond the range covered by the isotope ratios. Could, for example, the significantly higher source temperature derived for the heavier elements in the case of $Q / M$ fractionation (Figure 9) be associated with the inaccuracy of this extrapolation? At present, we cannot definitively answer such questions.

\subsection{Relation to Charge States Observed In Situ}

In impulsive SEP events, ${ }^{22} \mathrm{Ne} /{ }^{20} \mathrm{Ne}$ and $\mathrm{Fe} / \mathrm{O}$ (and $\mathrm{Fe} / \mathrm{C}$ ) are enhanced relative to their solar wind values. If the elemental and isotopic enhancements are organized as a power law of the form $(Q / M)^{\alpha}$, the observed enhancement of ${ }^{22} \mathrm{Ne} /{ }^{20} \mathrm{Ne}$ requires $\alpha<0$, which would imply that $\mathrm{Fe} / \mathrm{O}$ should decrease with increasing $Q_{\mathrm{Fe}}$ (assuming fixed values of $Q_{\mathrm{O}}$ and $\alpha$ ). However, statistical studies of the correlation between values of $\mathrm{Fe} / \mathrm{O}$ and $Q_{\mathrm{Fe}}^{\mathrm{obs}}$ measured below $1 \mathrm{MeV}$ nucleon $^{-1}$ in a number of impulsive events show $\mathrm{Fe} / \mathrm{O}$ increasing with increasing $Q_{\mathrm{Fe}}^{\mathrm{obs}}$ (Möbius et al. 2000; DiFabio et al. 2008). It is not necessarily the case that these two results are inconsistent. Additional factors that need to be taken into account include the following.

1. The $Q$ values that affect the charge and mass fractionation are those in the source material and not those observed in situ.

2. Although the enhancement of $\mathrm{Fe} / \mathrm{O}$ depends both on $Q_{\mathrm{Fe}}$ and on the strength of the fractionation, which we characterize by the parameter $\alpha$, values of $\alpha$ (as indicated, for example, by the enhancement of ${ }^{22} \mathrm{Ne} /{ }^{20} \mathrm{Ne}$ ) are unknown in the DiFabio et al. (2008) study, just as $Q_{\mathrm{Fe}}^{\text {obs }}$ is unknown in our investigation. The interpretation of the correlations that are found between $\mathrm{Fe} / \mathrm{O}$ and $Q_{\mathrm{Fe}}^{\text {obs }}$ when neglecting $\alpha$ or 
between $\mathrm{Fe} / \mathrm{O}$ and ${ }^{22} \mathrm{Ne} /{ }^{20} \mathrm{Ne}$ when neglecting $Q_{\mathrm{Fe}}$ could be altered if there is a correlation between charge state and strength of fractionation (as is the case in gradual events).

3. As shown by DiFabio et al. (2008), the statistical correlation between $\mathrm{Fe} / \mathrm{O}$ and $Q_{\mathrm{Fe}}^{\mathrm{obs}}$ in impulsive events decreases in significance with increasing energy, with a relatively strong correlation below $0.1 \mathrm{MeV}$ nucleon $^{-1}$ and essentially no correlation at $0.5 \mathrm{MeV}$ nucleon $^{-1}$, possibly reflecting the effect of collisional stripping that occurs over this energy interval.

4. Our assumption that acceleration, stripping, and fractionation can be treated as sequential processes may be an oversimplification of the actual situation. If the fractionated abundances observed below $1 \mathrm{MeV}$ nucleon $^{-1}$ actually represented an intermediate state in going from solar wind composition to the composition observed above $10 \mathrm{MeV}$ nucleon $^{-1}$, this would certainly be the case. A more specific model is needed to analyze these more complex situations.

5. It is not clear that the charge states and composition observed in situ below $1 \mathrm{MeV}$ nucleon $^{-1}$ should also represent the charge states that the particles observed at higher energies would have had at these energies as they were being accelerated. The release histories from the Sun and even the release mechanism might be different.

6 . It is conceivable that the relatively rare impulsive events that are observable above $10 \mathrm{MeV}$ nucleon $^{-1}$ may have a different origin than the events that are commonly investigated below $1 \mathrm{MeV}$ nucleon $^{-1}$, particularly in view of their similarity to the events studied by Cohen et al. (1999a, 1999b), as discussed in Section 4.3.

Nevertheless, the opposite senses of the correlations between our inferred values of $Q_{\mathrm{Fe}}$ and $\mathrm{Fe} / \mathrm{O}$, on the one hand, and between the DiFabio et al. (2008) in situ values of $Q_{\mathrm{Fe}}^{\mathrm{obs}}$ and Fe/O, on the other, do suggest that alternative fractionation laws should be considered, including ones in which abundance enhancements are an increasing function of $Q$.

\subsection{Alternative Fractionation Laws}

The assumption that the elemental fractionation depends on $Q / M$, while physically reasonable given that the electromagnetic interactions likely to cause the fractionation should depend on magnetic rigidity, is on less solid ground than the observation that the isotopic fractionation is a power law in the ratio of the masses. One would expect the same isotope fractionation if the general form of the fractionation were a power law in $F(Q) / M$ for any function of the charge states, $F(Q)$. To investigate some alternative fractionation laws, we considered a generalized version of Equation (1) in which the power-law dependences on $Q$ and $M$ are allowed to have different exponents:

$$
\mathcal{E}_{i}=Q_{i}^{\beta} / M_{i}^{\alpha}
$$

Using the value $\alpha=-15.0 \pm 0.5$ obtained from the isotope fractionation analysis, we have derived the combinations of $\beta$ and $Q_{\mathrm{Fe}}$ that are consistent with the observed Fe/C enhancement of $54 \pm 22$ (Table 1). The results of this analysis, in which we considered both positive and negative values of $\beta$, are shown as the narrow regions bounded by thick, solid curves in Figure 13. The vertical lines correspond to the cases $\beta=\alpha$ (long dashes), presented in the preceding sections, and $\beta=2 \alpha$ (short dashes), which represents fractionation as a power law in $Q^{2} / M$ and is

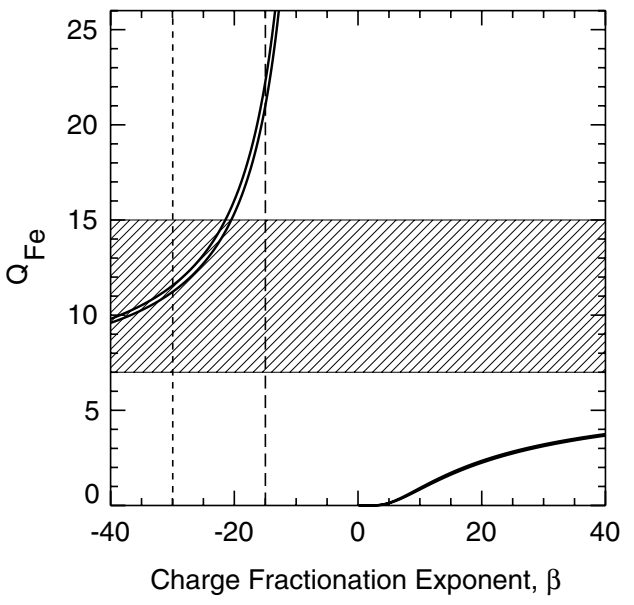

Figure 13. Combinations of values for $\beta$ and $Q_{\mathrm{Fe}}$ that are consistent with the observed enhancement of $\mathrm{Fe} / \mathrm{C}$ in the 2002 August 20 event (bands bounded by thick, solid curves) for a hypothetical fractionation law of the form $Q^{\beta} / M^{\alpha}$. Vertical lines correspond to fractionation as a power law in $Q / M$ (long dashes) and in $Q^{2} / M$ (short dashes). The exponent for the mass fractionation is held fixed at the value $\alpha=-15.0 \pm 0.5$ derived from the fit shown in Figure 6 . The typical range of solar wind Fe charge states is indicated by the hatched region.

discussed in the Appendix. The hatched region corresponds to the range of Fe charge states most commonly found in the solar wind, although values above and below this range are at times observed (see the bottom panel of Figure 8). Values ranging from this solar wind band up to fully stripped $\mathrm{Fe}\left(Q_{\mathrm{Fe}}=26\right)$ should be possible as the result of collisional stripping.

For positive values of $\beta$, the enhancement of $\mathrm{Fe} / \mathrm{C}$ observed in the 2002 August 20 event would require values of $Q_{\mathrm{Fe}}$ lower than those found in the solar wind. Furthermore, negative values of $\beta$ greater than $\sim-13$ are not possible because they would require $Q_{\mathrm{Fe}}$ greater than the charge of fully stripped Fe. Physically meaningful solutions are found only for $\beta \lesssim-13$. Of course, it is conceivable that fractionation laws other than that given in Equation (6) could reconcile the opposite correlations between of $Q_{\mathrm{Fe}}$ and $Q_{\mathrm{Fe}}^{\mathrm{obs}}$ with $\mathrm{Fe} / \mathrm{O}$.

At present, the apparent contradiction between elemental and isotopic fractionation studies on one hand and statistical studies correlating $Q_{\mathrm{Fe}}^{\mathrm{obs}}$ and $\mathrm{Fe} / \mathrm{O}$ on the other remains unresolved. It has also been noted (Leske et al. 2001) that a similar apparent contradiction occurs in the case of gradual SEP events.

The $Q$-dependences of the fractionation in both gradual and impulsive events and their possible dependence on energy clearly require further study. It would be of particular interest to compare inferred charge states derived from elemental and isotopic fractionation data obtained below $1 \mathrm{MeV}$ nucleon $^{-1}$ and above $10 \mathrm{MeV}$ nucleon $^{-1}$ with each other and with in situ measurements of charge states in the same events. The necessary data for such a study may be available for a few of the events observed during solar cycle 23 using the SIS, ULEIS, and SEPICA instruments on $A C E$ and/or the MAST and LICA instruments on SAMPEX.

\section{SUMMARY}

We have reported the results of detailed elemental and isotopic composition analysis of measurements made above $10 \mathrm{MeV}$ nucleon $^{-1}$ using the $A C E / \mathrm{SIS}$ instrument in the impulsive SEP event of 2002 August 20. Isotopes were found to be fractionated as a power law in the mass ratio, consistent with the previously reported correlation between the enhancements 
of ${ }^{22} \mathrm{Ne} /{ }^{20} \mathrm{Ne}$ and ${ }^{26} \mathrm{Mg} /{ }^{24} \mathrm{Mg}$ (Leske et al. 2007; Wiedenbeck et al. 2008). In the case of the 2002 August 20 event, the powerlaw exponent was found to be large, $\sim 15.0 \pm 0.5$, consistent with the established tendency toward significantly stronger mass fractionation in impulsive than in gradual SEP events. Elemental abundance enhancements relative to solar wind values were found to be characterized by a pattern that has been reported in a number of other impulsive SEP events at high energies (Wiedenbeck et al. 2008, 2009) in which the enhancement factor as a function of $Z$ has local maxima at $\mathrm{N}, \mathrm{Ne}$, and $\mathrm{Al}$, local minima at $\mathrm{O}$ and $\mathrm{S}$, and a plateau between $\mathrm{Ar}$ and $\mathrm{Ni}$.

We have assumed that the isotope fractionation as a power law in the mass ratio represents a special case of a general fractionation law that depends on $Q / M$, the quantity which, in conjunction with the energy per nucleon, determines the strength of interactions with electromagnetic fields. Applying a technique introduced by Cohen et al. (1999b) for the case of $Q / M$ fractionation and assuming that solar wind abundances provide an adequate approximation to the elemental and isotopic composition of the source population, we derived ionic charge states for a number of elements between $\mathrm{C}$ and $\mathrm{Ni}$.

We found charge states characterized by a series of step increases in the number of attached electrons, $Z-Q_{Z}$, occurring between $\mathrm{C}$ and $\mathrm{N}$, between $\mathrm{O}$ and $\mathrm{Ne}$, and between $\mathrm{S}$ and $\mathrm{Ar}$. This pattern, combined with the masses of the elements, was used to account for the structure found in the curve of elemental abundance enhancements versus $Z$. When we attempted to associate the charge states with an isothermal source plasma, we found a best-fit temperature $\sim 4 \mathrm{MK}$, but with significant deviations of some of the inferred charge states from the calculated dependence of $Q_{Z}$ on $Z$ at this temperature. The largest deviations occurred for $\mathrm{Fe}$ and $\mathrm{Ni}$, as previously found by Reames et al. (1994). The pattern of charge states derived for the 2002 August 20 event was found to be very similar to that previously derived by Cohen et al. (1999a) in a study of four events with comparable heavy-ion intensities and small but measurable enhancements of ${ }^{3} \mathrm{He} /{ }^{4} \mathrm{He}$, although in those events the fractionation exponents inferred from isotope measurements (Leske et al. 1999) were not as extreme.

The ionic charge states inferred from the elemental and isotopic fractionation should correspond to those present at the time the material was fractionated and need not be consistent with charge states observed in situ. To make further progress toward establishing the validity of this indirect method, we suggest a comparison of high-energy ( $>10 \mathrm{MeV}^{\text {nucleon }}{ }^{-1}$ ) and low-energy $\left(<1 \mathrm{MeV}\right.$ nucleon $\left.{ }^{-1}\right)$ composition including, as a minimum, ${ }^{22} \mathrm{Ne} /{ }^{20} \mathrm{Ne}$ and $\mathrm{Fe} / \mathrm{C}$, together with in situ charge state measurements in the low-energy range in a number of impulsive SEP events. If the validity of the inferred charge states can be confirmed, this technique provides a unique opportunity for probing physical conditions in the environment where the fractionation occurs.

We are grateful to J. Mazur for providing charge state estimates from $S A M P E X / \mathrm{LICA}, \mathrm{S}$. Krucker for providing information about the RHESSI X-ray imaging measurements in the 2002 August 20 event, A. Labrador for providing some unpublished charge-state data included in Figure 8, Y.-K. Ko for advice about the comparison with solar wind charge states, and G. Mason for providing the ULEIS data shown in Figure 12. In addition, we acknowledge useful discussions with E. Möbius, V. Petrosian, and J. Drake. This study also benefited from discussions at international team meetings sponsored by the Inter-

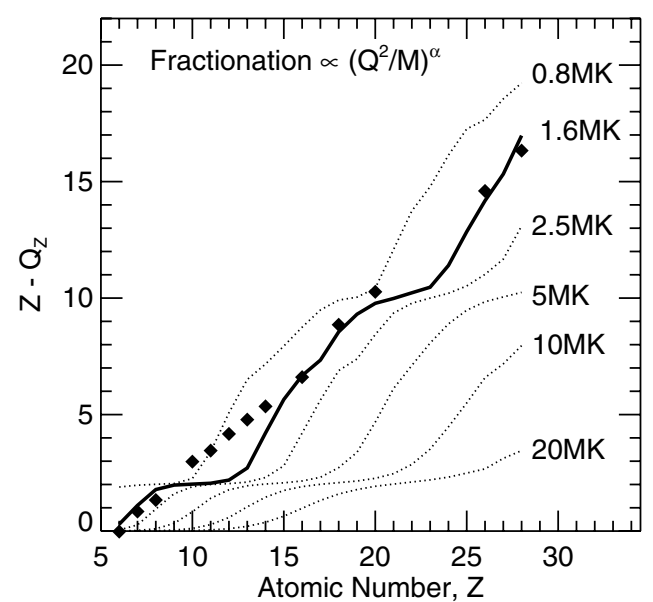

Figure 14. Mean charge states inferred assuming fractionation going as $\left(Q^{2} / M\right)^{\alpha}$ (points) compared with calculated equilibrium charge states as a function of temperature (Mazzotta et al. 1998). The heavy curve corresponds to the best-fit temperature of 1.6 MK. See the text for details.

national Space Science Institute (ISSI) in 2006 and 2007 and at the NSF-sponsored SHINE workshop in 2008. We thank E. Chollet and the anonymous referee for helpful comments on the manuscript. We gratefully acknowledge the $A C E /$ SWICS team for providing solar wind charge state data used in this study through the ACE Science Center Web site. This research was supported by NASA at Caltech (under grants NAG5-12929 and NNX08AI11G), JPL, and GSFC.

\section{APPENDIX}

\section{FRACTIONATION AS A POWER LAW IN $Q^{2} / M$}

As seen in Figure 13, a fractionation law going as a power law in $Q^{2} / M$ would yield a physically possible value of $Q_{\mathrm{Fe}} \simeq 11.4$ for the source material in the 2002 August 20 event. There is also some physical basis for considering the possibility that the fractionation depends on the parameter $Q^{2} / M$ since the rate of energy loss via Coulomb collisions during acceleration depends on this combination of mass and ionic charge (e.g., Mullan \& Levine 1981; Liu et al. 2006). In this Appendix, we examine the implications of this alternative fractionation law.

If one assumes that the fractionation goes as a power law in $Q^{k} / M$ for some constant $k$, then Equation (2) for the inferred charge states becomes

$Q_{Z}=Q_{Z_{0}}\left[\frac{M_{Z}}{M_{Z_{0}}}\left(\frac{\mathcal{E}_{Z}}{\mathcal{E}_{Z_{0}}}\right)^{1 / \alpha}\right]^{1 / k}$ (for $\left[Q^{k} / M\right]^{\alpha}$ fractionation).

Applying this result to the case of fractionation as a power law in $Q^{2} / M(k=2)$, we obtain the charge states shown as the points in Figure 14, again plotted in the form $Z-Q_{Z}$. Here, the number of electrons attached increases approximately uniformly at a rate of 0.73 electrons per unit increase in atomic number going from $\mathrm{C}$ to $\mathrm{Fe}$, with little or no indication of atomic shell effects.

The value of $Q_{\mathrm{Fe}}$ inferred in the 2002 August 20 event when $Q^{2} / M$-dependent fractionation is assumed (Figure 8, left-hand vertical band) is in the range typically found for Fe charge states in the solar wind and for low-energy SEPs observed in either impulsive or gradual events. Combined $Q_{\mathrm{Fe}}^{\mathrm{obs}}$ data from several impulsive events observed below $0.1 \mathrm{MeV}^{\text {nucleon }}{ }^{-1}$ using SOHO/STOF (Klecker et al. 2006b) yielded a value $\sim 12.5 \pm$ 1.0, similar to the result we obtain assuming fractionation 

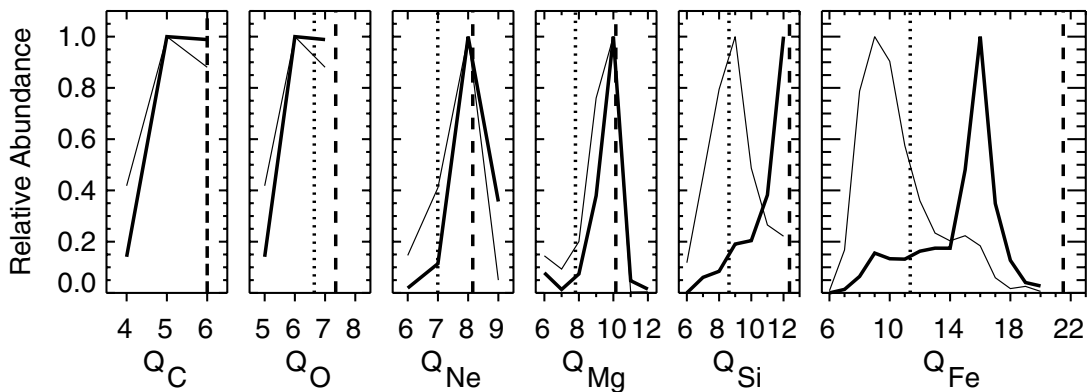

Figure 15. Comparison of inferred values of $Q_{Z}$ in the 2002 August 20 SEP event with solar wind charge state distributions from ACE/SWICS averaged over a 24 hr period centered on the start of the SEP event (thick solid line) and averaged over the year 2002 (thin solid line). The inferred values of $Q_{Z}$ have been normalized assuming $Q_{\mathrm{C}}=6.0$ (dashed line in the leftmost panel). Dotted (dashed) vertical lines show the SEP charge states derived assuming $Q^{2} / M(Q / M)$ fractionation.

depending on $Q^{2} / M$. These results are consistent with the interpretation favored by DiFabio et al. (2008) in which the SEPs are accelerated out of a source plasma having a temperature in the range 1.3-3 MK (see Section 4.1) and are progressively stripped to higher charge states during acceleration.

In Figure 14, we compare the mean charge states inferred assuming fractionation going as $\left(Q^{2} / M\right)^{\alpha}$ with charge states calculated for a plasma in thermal equilibrium (Mazzotta et al. 1998). The overall scaling of the inferred charge states was done as described in Section 4.1. We find a best-fit temperature $\sim 1.6 \mathrm{MK}$ and the best scaling of the $Q_{Z}$ values at that temperature results in $Q_{\mathrm{C}} \simeq 6.0$, which is higher than the value $\sim 5.7$ expected in a $1.6 \mathrm{MK}$ plasma. The minimum value of $\chi_{v}^{2}$ is very poor, as is clear from the comparison of the points in Figure 14 with the curve for $1.6 \mathrm{MK}$. Studies of charge state freeze-in temperatures for a variety of ions between $\mathrm{C}$ and $\mathrm{Fe}$ in slow solar wind (Aellig et al. 1998; Ko et al. 1999; Hefti et al. 2000) and also in fast solar wind (Ko et al. 1997, 1999) yield values in the range $\sim 1-2.5 \mathrm{MK}$, and most commonly less than $1.5 \mathrm{MK}$. The similarity of the best-fit temperature we infer for the SEP source material during $\left(Q^{2} / M\right)^{\alpha}$ fractionation to the solar wind temperatures could be taken to suggest that both solar wind and impulsive SEPs are being derived from similar populations of coronal material. However, the relatively featureless dependence of $Z-Q_{Z}$ versus $Z$, which is not consistent with structure found in the calculated curve for 1.6 MK, makes it seem unlikely that the elemental and isotopic abundances are actually derived by $Q^{2} / M$ fractionation of an isothermal source at this temperature.

It is also possible to directly compare the inferred SEP $Q_{Z}$ values with charge state distributions in the solar wind emanating from the same longitude on the Sun where the flare occurred by using data from $A C E /$ SWICS $^{4}$ (Gloeckler et al. 1998). In Figure 15, the relative abundances of the charge states contained in the SWICS data set $\left(\mathrm{C}^{+4}\right.$ through $\mathrm{C}^{+6}, \mathrm{O}^{+5}$ through $\mathrm{O}^{+8}, \mathrm{Ne}^{+6}$ through $\mathrm{Ne}^{+9}, \mathrm{Mg}^{+6}$ through $\mathrm{Mg}^{+12}, \mathrm{Si}^{+6}$ through $\mathrm{Si}^{+12}$, and $\mathrm{Fe}^{+6}$ through $\mathrm{Fe}^{+20}$ ) are shown averaged over a $24 \mathrm{hr}$ period centered on the onset of the SEP event (thick solid lines). Also shown (thin solid lines) are long-term average solar wind charge state distributions covering the year 2002. The shorterterm sample comes from the early part of a $\sim 4-5$ day period when the solar wind charge states increased and subsequently returned to more-typical values, which are similar to those in the full-year average.

From the surface at which the magnetic field becomes frozen into the solar wind plasma at a few solar radii, the plasma

\footnotetext{
4 www.srl.caltech.edu/ACE/ASC/level2/lv12DATA_SWICS-SWIMS.html
}

should come from the same location as the energetic particles but with release times from the Sun that differ by a few days. Active region magnetic fields often undergo significant nonradial expansion between the photosphere and the source surface (e.g., Liewer et al. 2004), so one cannot necessarily assume that the plasma conditions observed at $1 \mathrm{AU}$ correspond to those near the flare site. However, the active region from which the 2002 August 20 event originated was at low heliolatitude and reasonably well connected to $A C E$ (S10W38), suggesting that there may be such a correspondence for this event. Cane \& Richardson (2003) identified the plasma observed at $A C E$ around the time of the 2002 August 20 event as being associated with an interplanetary CME. The inferred charge states derived assuming fractionation $\propto\left(Q^{2} / M\right)^{\alpha}$ and normalized using the assumed value $Q_{\mathrm{C}}=6.0$ (dashed line in left-hand panel) are shown by the vertical dotted lines. The inferred $Q_{Z}$ values all fall within the ranges covered by the solar wind distributions. From this comparison it is not clear whether the hot coronal material indicated by the elevated solar wind charge states around the time of the 2002 August 20 event may have played a role in causing SEP abundance fractionation observed in this event.

If one compares these same solar wind charge state distributions with the SEP source values inferred assuming $Q / M$ fractionation (vertical dashed lines), the agreement with the modal values in the solar wind $24 \mathrm{hr}$ distribution is typically better than for $Q^{2} / M$ fractionation, with the important exception of $Q_{\mathrm{Fe}}$ where the SEP value $\sim 21-22$ in the $Q / M$ fractionation case is significantly greater than the solar wind value of $\sim 16$.

In our interpretation of the charge states inferred assuming fractionation depending on $Q / M$ (Section 4.2), we argued that stripping prior to fractionation might produce the high value of $Q_{\mathrm{Fe}}$ and narrow the charge state distributions so that they would be dominated by a single charge state. This narrowing was important both for avoiding large contributions from low$Q$, low-abundance charge states due to the strong $Q$-dependence of the fractionation law and also for explaining the presence of integer steps in the pattern of inferred charge states. In the case of fractionation dependent on $Q^{2} / M$, the mean charge states on which the fractionation would be acting are found to be close to the solar wind values, leaving little room for additional stripping which might be capable of narrowing the distributions. However, if relatively broad charge state distributions such as that found for $\mathrm{Fe}$ in the solar wind (Figure 15) were subjected to fractionation with a strong $Q$-dependence $\left(\propto Q^{-30}\right)$ such as that derived for the 2002 August 20 event, the enhancement of low charge states would be enormous and would cause the elemental abundances in the fractionated material to have a very 
strong dependence on the abundances of the lowest charge states present in the source material for each element. Thus, it seems unlikely that the impulsive SEP abundance enhancements are attributable to fractionation as a power law in $Q^{2} / M$.

\section{REFERENCES}

Aellig, M. R., et al. 1998, J. Geophys. Res., 103, 17215

Aschwanden, M. J. 2002, Space Sci. Rev., 101, 1

Barghouty, A. F., \& Mewaldt, R. A. 1999, ApJ, 520, L127

Barghouty, A. F., \& Mewaldt, R. A. 2000, in AIP Conf. Proc. 528, Acceleration and Transport of Energetic Particles Observed in the Heliosphere, ed. R. A. Mewaldt et al. (Melville, NY: AIP), 71

Bochsler, P. 2007, A\&AR, 14, 1

Breneman, H. H., \& Stone, E. C. 1985, ApJ, 299, L57

Cane, H. V., \& Richardson, I. G. 2003, J. Geophys. Res. (Space Phys.), 108, 1156

Cane, H. V., von Rosenvinge, T. T., Cohen, C. M. S., \& Mewaldt, R. A. 2003, Geophys. Res. Lett., 30, 8017

Cohen, C. M. S., Mewaldt, R. A., Leske, R. A., Cummings, A. C., Stone, E. C., Wiedenbeck, M. E., Christian, E. R., \& von Rosenvinge, T. T. 1999a, Geophys. Res. Lett., 26, 2697

Cohen, C. M. S., et al. 1999b, Geophys. Res. Lett., 26, 149

Dennis, B. R., Hudson, H. S., \& Krucker, S. 2007, in The High Energy Solar Corona: Waves, Eruptions, Particles (Lecture Notes in Physics, Vol. 725), ed. K.-L. Klein \& A. L. MacKinnon (Berlin: Springer), 33

DiFabio, R., Guo, Z., Möbius, E., Klecker, B., Kucharek, H., Mason, G. M., \& Popecki, M. 2008, ApJ, 687, 623

Drake, J. F., Cassak, P. A., Shay, M. A., Swisdak, M., \& Quataert, E. 2009, ApJ, 700, L16

Drake, J. F., Swisdak, M., Che, H., \& Shay, M. A. 2006, Nature, 443, 553

Dwyer, J. R., Mason, G. M., Mazur, J. E., Gold, R. E., Krimigis, S. M., Möbius, E., \& Popecki, M. 2001, ApJ, 563, 403

Feldman, U. 1996, Phys. Plasmas, 3, 3203

Garrard, T. L., \& Stone, E. C. 1994, Adv. Space Res., 14, 589

Gloeckler, G., et al. 1998, Space Sci. Rev., 86, 497

Guo, Z., Möbius, E., \& Popecki, M. 2008, ACE News 116, www.srl.caltech.edu/ACE/ASC/level2/lv12DATA_SWICS-SWIMS.html

Hefti, S., Grünwaldt, H., Bochsler, P., \& Aellig, M. R. 2000, J. Geophys. Res., 105,10527

Karrer, R., Bochsler, P., Giammanco, C., Ipavich, F. M., Paquette, J. A., \& Wurz, P. 2007, Space Sci. Rev., 130, 317

Kartavykh, Y. Y., Dröge, W., Klecker, B., Kocharov, L., Kovaltsov, G. A., \& Möbius, E. 2008, ApJ, 681, 1653

Kartavykh, J. J., Dröge, W., Kovaltsov, G. A., \& Ostryakov, V. M. 2005, Sol. Phys., 227, 123

Klecker, B., Hovestadt, D., Scholer, M., Gloeckler, G., Ipavich, F. M., Fan, C. Y., \& Fisk, L. A. 1984, ApJ, 281, 458

Klecker, B., Möbius, E., \& Popecki, M. A. 2006a, Space Sci. Rev., 124, 289

Klecker, B., Möbius, E., \& Popecki, M. A. 2007, Space Sci. Rev., 130, 273

Klecker, B., Möbius, E., Popecki, M. A., Kistler, L. M., Kucharek, H., \& Hilchenbach, M. 2005, in Solar Wind 11/SOHO 16, Connecting Sun and Heliosphere, ed. B. Fleck \& T. H. Zurbuchen (ESA Special Publication, Vol. 592; Noordwijk: ESA), 77

Klecker, B., Möbius, E., Popecki, M. A., Kistler, L. M., Kucharek, H., \& Hilchenbach, M. 2006b, Adv. Space Res., 38, 493

Klecker, B., et al. 2006c, Space Sci. Rev., 123, 217

Ko, Y.-K., Fisk, L. A., Geiss, J., Gloeckler, G., \& Guhathakurta, M. 1997, Sol. Phys., 171, 345

Ko, Y.-K., Gloeckler, G., Cohen, C. M. S., \& Galvin, A. B. 1999, J. Geophys. Res., 104, 17005

Kocharov, L., Kovaltsov, G. A., Torsti, J., \& Ostryakov, V. M. 2000, A\&A, 357, 716

Koeten, M., Wimmer-Schweingruber, R. F., Rodde, R., Berger, L., Gloeckler, G., \& Zurbuchen, T. H. 2008, in AGU Fall Meeting Abstracts (Washington, DC: AGU), A1570

Labrador, A. W., Leske, R. A., Mewaldt, R. A., Stone, E. C., \& von Rosenvinge, T. T. 2003, in Proc. 28th ICRC (Tsukuba), 6, 3269

Lepri, S. T., \& Zurbuchen, T. H. 2004, J. Geophys. Res. (Space Phys.), 109, 1112
Lepri, S. T., Zurbuchen, T. H., Fisk, L. A., Richardson, I. G., Cane, H. V., \& Gloeckler, G. 2001, J. Geophys. Res., 106, 29231

Leske, R. A., Mewaldt, R. A., Cohen, C. M. S., Cummings, A. C., Stone, E. C., Wiedenbeck, M. E., Christian, E. R., \& von Rosenvinge, T. T. 1999, Geophys. Res. Lett., 26, 2693

Leske, R. A., Mewaldt, R. A., Cohen, C. M. S., Cummings, A. C., Stone, E. C., Wiedenbeck, M. E., \& von Rosenvinge, T. T. 2007, Space Sci. Rev., 130, 195

Leske, R. A., Mewaldt, R. A., Cummings, A. C., Stone, E. C., \& von Rosenvinge, T. T. 2001, in AIP Conf. Proc. 598, Solar and Galactic Composition, ed. R. F. Wimmer-Schweingruber (Melville, NY: AIP), 171

Leske, R. A., Wiedenbeck, M. E., Cohen, C. M. S., Mewaldt, R. A., Cummings, A. C., Stone, E. C., \& von Rosenvinge, T. T. 2003, in Proc. 28th ICRC (Tsukuba), 6, 3253

Liewer, P. C., Neugebauer, M., \& Zurbuchen, T. 2004, Sol. Phys., 223, 209

Liu, S., Petrosian, V., \& Mason, G. M. 2006, ApJ, 636, 462

Lodders, K. 2003, ApJ, 591, 1220

Luhn, A., Klecker, B., Hovestadt, D., \& Möbius, E. 1987, ApJ, 317, 951

Ma Sung, L. S., Gloeckler, G., Fan, C. Y., \& Hovestadt, D. 1981, ApJ, 245, L45

Mason, G. M. 2007, Space Sci. Rev., 130, 231

Mason, G. M., Mazur, J. E., \& Dwyer, J. R. 2002a, ApJ, 565, L51

Mason, G. M., Mazur, J. E., Dwyer, J. R., Jokipii, J. R., Gold, R. E., \& Krimigis, S. M. 2004, ApJ, 606, 555

Mason, G. M., Reames, D. V., von Rosenvinge, T. T., Klecker, B., \& Hovestadt, D. 1986, ApJ, 303, 849

Mason, G. M., et al. 2002b, ApJ, 574, 1039

Mazur, J. E., Mason, G. M., Looper, M. D., Leske, R. A., \& Mewaldt, R. A. 1999, Geophys. Res. Lett., 26, 173

Mazzotta, P., Mazzitelli, G., Colafrancesco, S., \& Vittorio, N. 1998, A\&AS, 133,403

Möbius, E., Hovestadt, D., Klecker, B., \& Gloeckler, G. 1980, ApJ, 238, 768

Möbius, E., et al. 1998, Space Sci. Rev., 86, 449

Möbius, E., et al. 2000, in AIP Conf. Proc. 528, Acceleration and Transport of Energetic Particles Observed in the Heliosphere, ed. R. A. Mewaldt et al. (Melville, NY: AIP), 131

Mullan, D. J. 1983, ApJ, 268, 385

Mullan, D. J., \& Levine, R. H. 1981, ApJS, 47, 87

Petrosian, V. 2008, arXiv:0808.1757

Petrosian, V., Jiang, Y. W., Liu, S., Ho, G. C., \& Mason, G. M. 2009, ApJ, 701, 1

Reames, D. V. 1995a, Adv. Space Res., 15, 41

Reames, D. V. 1995b, Rev. Geophys., 33, 585

Reames, D. V. 1999, Space Sci. Rev., 90, 413

Reames, D. V., Meyer, J. P., \& von Rosenvinge, T. T. 1994, ApJS, 90, 649

Reames, D. V., \& Ng, C. K. 2004, ApJ, 610, 510

Sciambi, R. K., Gloeckler, G., Fan, C. Y., \& Hovestadt, D. 1977, ApJ, 214, 316

Scott, P. C., Asplund, M., Grevesse, N., \& Sauval, A. J. 2006, A\&A, 456, 675

Slocum, P. L., et al. 2003, ApJ, 594, 592

Stone, E. C., et al. 1998a, Space Sci. Rev., 86, 357

Stone, E. C., et al. 1998b, Space Sci. Rev., 86, 285

Tylka, A. J., Cohen, C. M. S., Dietrich, W. F., Maclennan, C. G., McGuire, R. E., Ng, C. K., \& Reames, D. V. 2001, ApJ, 558, L59

von Rosenvinge, T., \& Cane, H. V. 2006, in Solar Eruptions and Energetic Particles (AGU Geophysical Monograph 165), ed. N. Gopalswamy et al., 103

Wiedenbeck, M. E., Cohen, C. M. S., Leske, R. A., Mewaldt, R. A., Cummings, A. C., Stone, E. C., \& von Rosenvinge, T. T. 2009, in Proc. 31st ICRC (Lodz), paper 544

Wiedenbeck, M. E., Leske, R. A., Cohen, C. M. S., Cummings, A. C., Mewaldt, R. A., Stone, E. C., \& von Rosenvinge, T. T. 2003, in Proc. 28th ICRC (Tsukuba), 6, 3245

Wiedenbeck, M. E., Leske, R. A., Cohen, C. M. S., Cummings, A. C., Mewaldt, R. A., Stone, E. C., \& von Rosenvinge, T. T. 2008, in AIP Conf. Proc. 1039, Particle Acceleration and Transport in the Heliosphere and Beyond, ed. G. Li et al. (Melville, NY: AIP), 149

Woods, P. M. 2009, arXiv:0901:4513

Woods, P. M., \& Willacy, K. 2009, ApJ, 693, 1360

Zhang, T. X., Wang, J. X., \& Tan, A. 2005, J. Geophys. Res. (Space Phys.), 110, 12111 